\title{
2. THE MIOCENE/PLIOCENE BOUNDARY IN THE EASTERN MEDITERRANEAN: RESULTS FROM SITES 967 AND 9691
}

\author{
Silvia Spezzaferri, ${ }^{2}$ Maria B. Cita, ${ }^{3}$ and Judith A. McKenzie $^{2}$
}

\begin{abstract}
Continuous sequences developed across the Miocene/Pliocene boundary were cored during Ocean Drilling Project (ODP) Leg 160 at Hole 967A, located on the base of the northern slope of the Eratosthenes Seamount, and at Hole 969B, some $700 \mathrm{~km}$ to the west of the previous location, on the inner plateau of the Mediterranean Ridge south of Crete. Multidisciplinary investigations, including quantitative and/or qualitative study of planktonic and benthic foraminifers and ostracodes and oxygen and carbon isotope analyses of these microfossils, provide new information on the paleoceanographic conditions during the latest Miocene (Messinian) and the re-establishment of deep marine conditions after the Messinian Salinity Crisis with the re-colonization of the Eastern Mediterranean Sea in the earliest Pliocene (Zanclean).

At Hole 967A, Zanclean pelagic oozes and/or hemipelagic marls overlie an upper Messinian brecciated carbonate sequence. At this site, the identification of the Miocene/Pliocene boundary between 119.1 and 119.4 mbsf, coincides with the lower boundary of the lithostratigraphic Unit II, where there is a shift from a high content of inorganic and non-marine calcite to a high content of biogenic calcite typical of a marine pelagic ooze. The presence of Cyprideis pannonica associated upward with Paratethyan ostracodes reveals that the upper Messinian sequence is complete. The stable isotope composition of $C$. pannonica indicates that this crustacean lived in a Lago Mare environment characterized by brackish water, distinctly different from the marine environment. At Hole 969B, the identification of the Miocene/Pliocene boundary coincides with a marked lithologic change between 97.1 and 97.2 mbsf. The marine sediments directly overlie latest Messinian Lago Mare facies deposits comprising dark gray clay. The presence of planktonic foraminifers associated with brackish water ostracodes in Hole 967A, and with the gray clay in Hole 969B, may indicate temporary incursions of Atlantic water into a fully Lago Mare environment. This interpretation is also supported by the trends in the isotopic composition of the ostracodes and planktonic foraminifers.

After the Messinian Salinity Crisis, benthic foraminifers slowly repopulated the Eastern Mediterranean seafloor during the Sphaeroidinellopsis acme event (Biozone MP11). The gradual ingression of typical deep Atlantic benthic foraminifers, Parrelloides bradyi and P. robertsonianus, and the presence of Globorotalia menardii (planktonic foraminifer) coincident with the boundary between the biozones MPI1 and MP12 indicate that an efficient connection between the Atlantic Ocean and the Eastern Mediterranean Sea was re-established in this interval.
\end{abstract}

\section{INTRODUCTION}

The paleogeography and paleoceanography of the Mediterranean experienced drastic changes during the latest Miocene (the latest part of the Messinian stage) when this marginal sea lost its connection with the Atlantic Ocean and became temporarily isolated as a result of the interaction of plate motions and glacio-eustatic sea-level changes (Ryan, Hsü, et al., 1973; Hsü, Montadert, et al., 1978). This isolation resulted in a "salinity crisis" of the Mediterranean, whereby the world's ocean lost $6 \%$ of its salinity because approximately one million cubic kilometers of evaporites were deposited on the floor of the deep Mediterranean basins (Hsü et al., 1973; Ryan, 1973).

The earliest Pliocene transgression (Zanclean stage) documents the re-establishment of open marine conditions in the Mediterranean. This drastic change is observed in both deep-sea settings and land exposures with carbonate pelagic oozes directly overlying Lago Mare deposits, which are characteristic for the latest post-evaporitic Messinian. Sedimentary successions across the Miocene/Pliocene boundary were encountered during Leg 160 in the Eastern Mediterranean at Hole 967A on the Eratosthenes Seamount and Site 969 on

${ }^{1}$ Robertson, A.H.F., Emeis, K.-C., Richter, C., and Camerlenghi, A. (Eds.), 1998. Proc. ODP, Sci. Results, 160: College Station, TX (Ocean Drilling Program).

${ }^{2}$ Geologisches Institut, ETH-Zentrum, Sonneggstrasse 5, 8092, Zurich, Switzerland. silvia@erdw.ethz.ch

${ }^{3}$ Dipartimento di Scienze della Terra, Universitá di Milano, Via Mangiagalli 34, 20133, Milano, Italy. the Mediterranean Ridge (Emeis, Robertson, Richter, et al., 1996; Fig. 1).

Previous studies of the Miocene/Pliocene boundary and the significance of the Pliocene transgression after the termination of the Messinian Salinity Crisis date back to the first drilling campaign in the Mediterranean during Deep Sea Drilling Program (DSDP) Leg

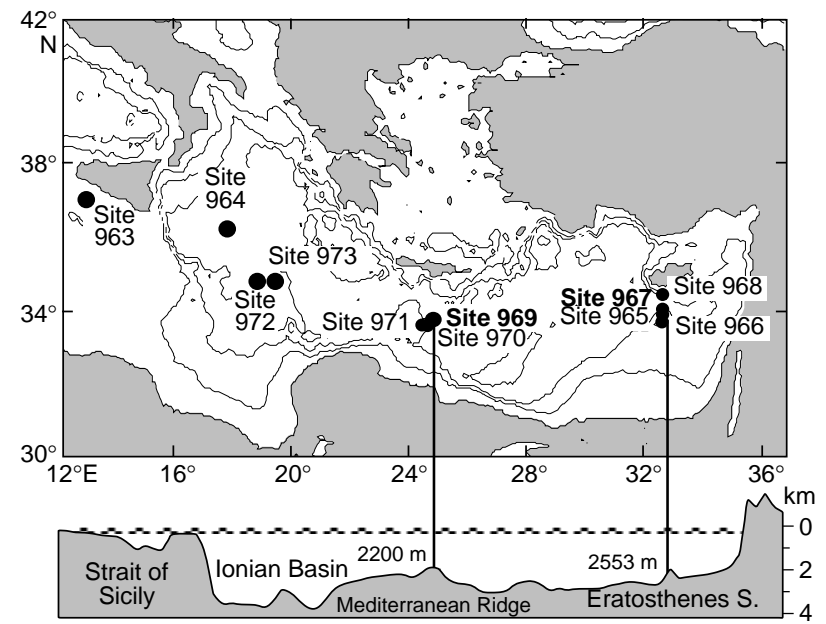

Figure 1. Map of the Eastern Mediterranean showing the locations of Sites 963-973. The depth positions of Sites 967 and 969 are indicated on the topographic profile shown in the inset. 
13, and subsequent land studies in Sicily (Ryan, Hsü, et al., 1973; Cita and Ryan, 1973; Cita, 1973b, 1975b; Cita and Gartner, 1973). The first paleomagnetic calibration of the Miocene/Pliocene boundary, providing precise timing of the event, was obtained by Zijderweld et al. (1986) in the Singa Section from Calabria. Subsequent high-resolution stratigraphy research carried out in Sicily and Calabria (Hilgen, 1991; Hilgen and Langereis, 1993) and in the Tyrrhenian ODP Site 652 (McKenzie and Sprovieri, 1990; McKenzie at al., $1988,1990)$ indicated that the boundary is isochronous on land (Eraclea Minoa, Rossello Composite, Singa Composite) and in the deep sea, at least in the central Mediterranean. The Miocene/Pliocene boundary as defined by Cita (1975a) at the base of the Zanclean at Capo Rossello is not yet formalized in a strictly chronostratigraphic sense. A global stratotype section and point (GSSP) has been recently proposed for a section in Morocco (Benson and Hodell, 1994), which has the Miocene/Pliocene boundary located in a position older than has been consistently used in the last 20 years.

The present study was undertaken using a multidisciplinary approach to investigate the sedimentary sequences obtained from the newest ODP drill sites in the Eastern Mediterranean. We propose to evaluate the stratigraphic events across the Miocene/Pliocene boundary in this region and compare them with sequences from the Western Mediterranean. Ultimately, the goal is to test the synchroneity of the events on a basin-wide scale.

\section{MATERIALS AND METHODS}

High-resolution sampling of the sediments from Holes 967A (Fig. 2) and 969E was done for this study aboard the JOIDES Resolution at closely spaced intervals of approximately $10-50 \mathrm{~cm}$ throughout the latest Miocene Lago Mare sequence and the MPl1 and MPl2 Zones. Hole 969B was sampled postcruise at the Bremen Core Repository (Fig. 3).

The sample volume was $\sim 5-10 \mathrm{~cm}^{3}$. Samples were soaked in distilled water, washed under running water through $40-$ to $150-\mu \mathrm{m}$, 151- to $250-\mu \mathrm{m}$, and $>250-\mu \mathrm{m}$ sieves. The three size fractions obtained were then dried at room temperature and weighed to determine eventual sorting and/or degree of dissolution (Premoli Silva et al., 1993; Spezzaferri, 1995). Samples were then re-assembled and drysieved through the $125-\mu \mathrm{m}$ mesh sieve for quantitative study. This was done to facilitate the comparison with previous studies (McKenzie et al., 1990: Sprovieri, 1993). Three hundred specimens of planktonic foraminifers were counted for each sample for the interval spanning the MP11 to MP12 biozones. No planktonic foraminifers were counted for Hole 969E where the oldest part of the Zanclean was not observed.

The calcium carbonate content was measured in bulk samples using a Dichter-Freeling calcimeter. Three to ten specimens of Oridorsalis stellatus and 20 to 30 specimens of Globigerinoides obliquus were picked from each sample for stable isotope analysis. Oxygen and carbon isotopes were measured using a PRISM Mass Spectrometer in the Stable Isotope Laboratory of the Geological Institute, ETH-Zurich. The isotope data, corrected following the procedure of Craig (1957), modified for a triple collector and relative to the international standard Pee Dee Belemnite (PDB), together with the data of weight of size fractions, percent abundance of planktonic foraminifers and carbonate content as well as the depth below seafloor (mbsf) and the revised composite depth (rmcd), are given in Tables 1-3.

\section{BIOSTRATIGRAPHIC RESULTS}

\section{Site 967 (Eratosthenes Seamount)}

Although Site 967 on the Eratosthenes Seamount was planned for tectonic objectives only, it proved to be an exceptional location for studying the Miocene/Pliocene boundary problem. Hole 967A is located on the lower northern slope of the Eratosthenes Seamount $\left(34^{\mathrm{O}} 04.098 \mathrm{~N}, 32^{\circ} 43.523^{\mathrm{E}} \mathrm{E}\right.$, water depth of $2553 \mathrm{~m}$, penetration of $141.3 \mathrm{~m}$ ). The advanced piston corer (APC) was used down to 123.3 mbsf. The extended core barrel (XCB) was used down to $141.3 \mathrm{mbsf}$. Recovery throughout was close to $100 \%$. The upper part of the sedimentary sequence comprises about $100 \mathrm{~m}$ of Pliocene-Quaternary nannofossil oozes and nannofossil clay with numerous sapropels. The lower part comprises slightly brecciated and indurated carbonates.

The investigated interval includes Core 160-967A-13H downward. In the Leg 160 Initial Reports volume (Emeis, Robertson, Richter, et al., 1996), the sedimentary section is described in the barrel sheets as lower Pliocene until the upper part of Core 160-967A$15 \mathrm{X}$, but this is not the case. Strong coring disturbance is notable in Core 160-967A-13H starting from Section 160-967A-13H-4 (see Fig. 2 where the locations of the 29 investigated samples are shown). No typical Pliocene age assemblages are recorded lower than Section 160-967A-13H-3.

Planktonic and benthic foraminiferal assemblages, plus ostracodes, indicate a variety of ages and environments. Of special interest is the recorded occurrence of the so-called Lago Mare biofacies typical of the latest post-evaporitic Messinian. Notably, at this location no Messinian evaporites were cored. The pore-water profiles of salinity, chlorite, and sodium, however, reach values almost twice those of seawater, suggesting that evaporites or an evaporite brine may be present deeper (>150 mbsf) downhole (Emeis, Robertson, Richter, et al., 1996). This interpretation is also supported by the downhole logging data (Major et al., Chap. 38, this volume). Age determination for the lower Pliocene sequences identified in this study are based on planktonic foraminiferal zonal schemes proposed by Cita (1975b) and amended by Sprovieri (1992, 1993).

\section{Planktonic Foraminifers}

Planktonic foraminifers range in abundance from absent in the lower part of the section to dominant. They consist of diversified and poorly to well-preserved lower Pliocene (Zanclean) faunas.

Zone MPl2 is identified from Sample 160-967A-12H-CC, 42-44 $\mathrm{cm}$, to $13 \mathrm{H}-2,19-21 \mathrm{~cm}$. Planktonic foraminiferal assemblages include common to abundant Orbulina universa, Globigerinoides obliquus, G. quadrilobatus, G. sacculifer, G. trilobus, Globorotalia margaritae, G. scitula, Globigerina falconensis, Globigerinita juvenilis, G. glutinata, Neogloboquadrina acostaensis dextral and sinistral, Zeaglobigerina woodi, Turborotalita quinqueloba, and the $Z$. nepenthes group. Minor components are Globigerina bulloides, Globigerinoides ruber, Sphaeroidinellopsis group (which includes $S$. seminulina, S. subdehiscens, and S. kochi), and Zeaglobigerina decoraperta. Specimens belonging to the Globorotalia menardii group were observed across the MP11/MP12 zonal boundary in Samples 160-967A-13H-2, 19-21 cm, and 13H-2, 135-37 cm (Pl. 1, Fig. 12).

Zone MP11 is identified from Sample 160-967A-13H-2, 35-37 $\mathrm{cm}$, to $13 \mathrm{H}-4,78-80 \mathrm{~cm}$, with a total thickness of $3.43 \mathrm{~m}$. Planktonic foraminiferal assemblages are similar to those observed in Zone MP12, except for the absence of G. margaritae. The Sphaeroidinellopsis acme event is identified from Sample 160-967A-13H-3, 25-27 $\mathrm{cm}$, to $13 \mathrm{H}-3,113-115 \mathrm{~cm}$ (Table 4).

From Samples 160-967A-13H-4, 25-27 cm, to $13 \mathrm{H}-4,78-80 \mathrm{~cm}$, rare large-sized planktonic foraminifers occur together with a dwarf fauna predominantly composed of $T$. quinqueloba. This assemblage is interpreted as the lowermost Zanclean assemblage, predating the Sphaeroidinellopsis acme event.

Messinian sediments were recovered from Sample 160-967A$13 \mathrm{H}-4,109-111 \mathrm{~cm}$, to $16 \mathrm{X}-1,84-86 \mathrm{~cm}$. These sediments are characterized by mixed Eocene, Oligocene, and middle Miocene planktonic foraminiferal assemblages (Table 5). Reworked specimens in- 


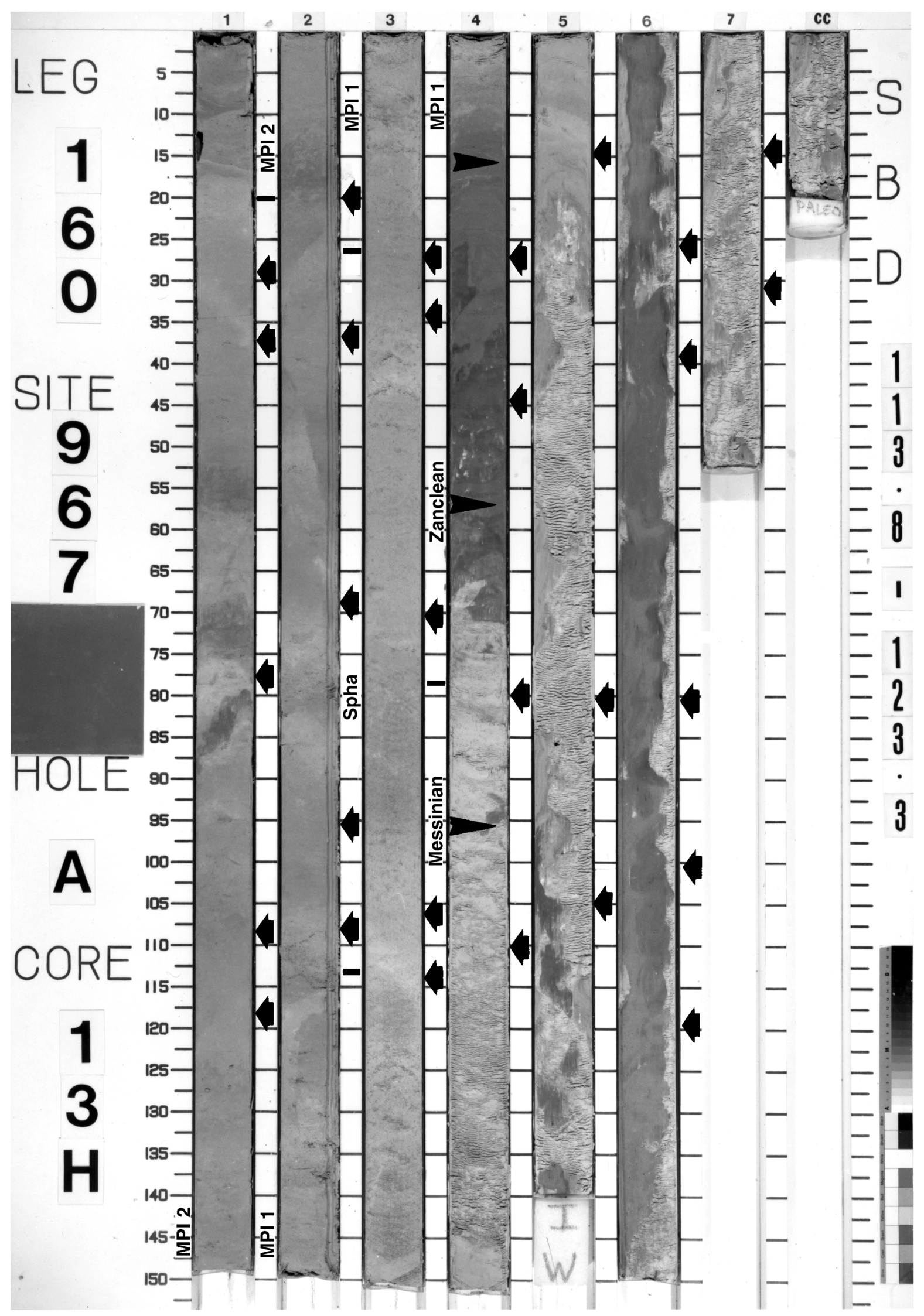

Figure 2. Photograph of Core 160-967A-13H, showing the location of the 29 investigated samples. 


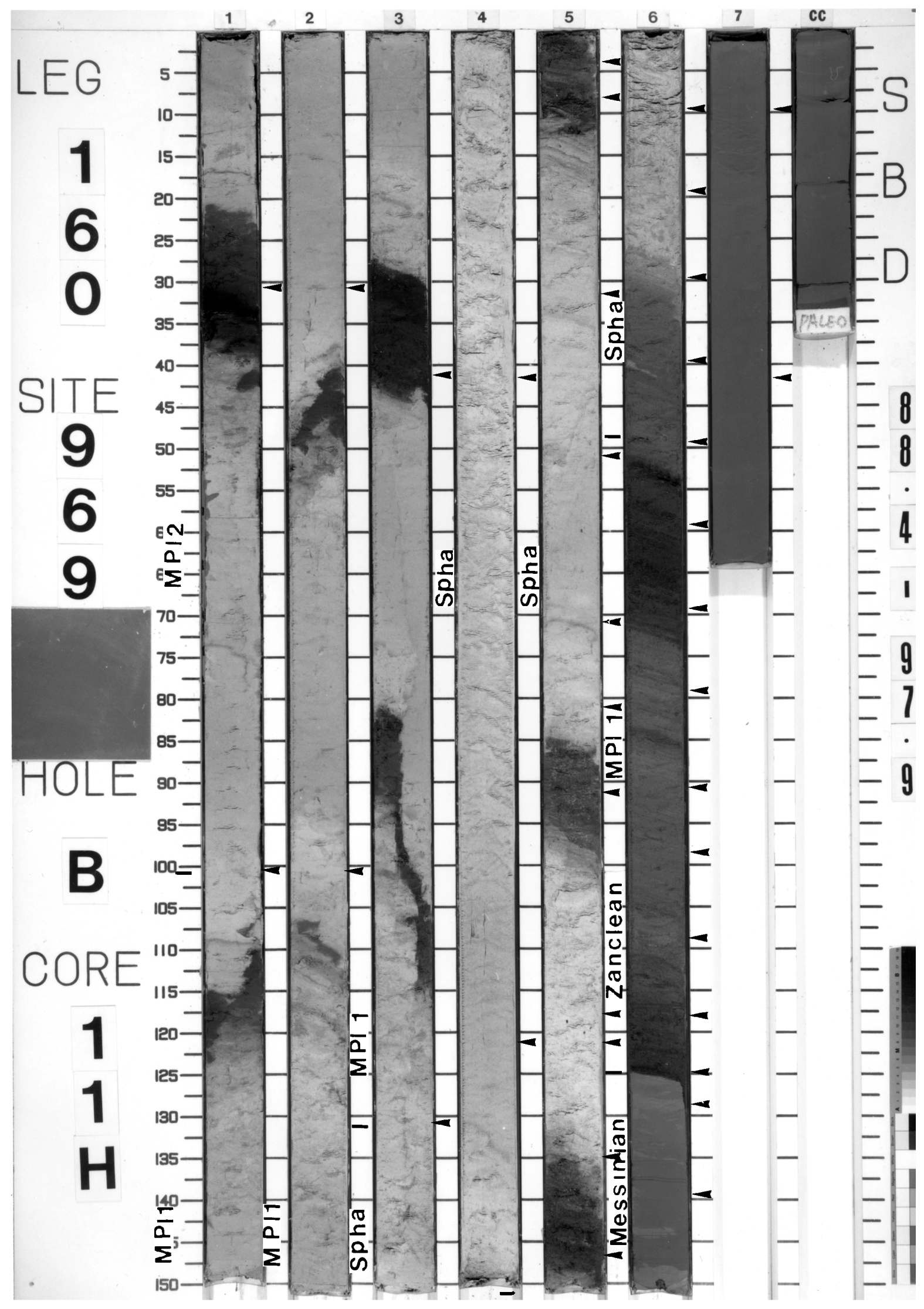

Figure 3. Photograph of Core 160-969B-11H, showing the location of the 36 investigated samples. 
Table 1. Abundance of size fractions (>151 $\mu \mathrm{m}, 40-151 \mu \mathrm{m}$, and $<40 \mu \mathrm{m})$, calcium carbonate content, and stable isotopes of $G$. obliquus, $O$. stellatus, and $C$. pannonica plotted vs. mbsf and rmed for Hole $967 \mathrm{~A}$.

\begin{tabular}{|c|c|c|c|c|c|c|c|c|c|c|c|c|}
\hline \multirow[b]{2}{*}{$\begin{array}{l}\text { Core, section, } \\
\text { interval }(\mathrm{cm})\end{array}$} & \multicolumn{2}{|c|}{ Depth } & \multirow[b]{2}{*}{$\begin{array}{c}\mathrm{CaCO}_{3} \\
(\mathrm{wt} \%)\end{array}$} & \multicolumn{3}{|c|}{ Size fraction } & \multicolumn{2}{|c|}{ Planktonic foraminifers } & \multicolumn{2}{|c|}{ Benthic foraminifers } & \multicolumn{2}{|c|}{ Ostracodes C. pannonica } \\
\hline & (mbsf) & (rmcd) & & $>151 \mu \mathrm{m}$ & $\begin{array}{c}40-151 \\
\mu \mathrm{m}\end{array}$ & $<40 \mu \mathrm{m}$ & $\delta^{13} \mathrm{C}$ & $\delta^{18} \mathrm{O}$ & $\delta^{13} \mathrm{C}$ & $\delta^{18} \mathrm{O}$ & $\delta^{13} \mathrm{C}$ & $\delta^{18} \mathrm{O}$ \\
\hline \multicolumn{13}{|l|}{ 160-967A- } \\
\hline $12 \mathrm{H}-\mathrm{CC}$ & 113.450 & 120.310 & - & - & - & - & 0.995 & -0.527 & -0.674 & 1.616 & - & - \\
\hline $13 \mathrm{H}-1,27-29$ & 114.070 & 122.960 & 55.260 & 1.980 & 1.630 & 0.940 & 1.259 & 0.109 & -0.982 & 1.821 & - & - \\
\hline $13 \mathrm{H}-1,35-37$ & 114.150 & 123.060 & 55.380 & 1.903 & 1.130 & 0.815 & 1.166 & 0.259 & - & - & - & - \\
\hline $13 \mathrm{H}-1,77-79$ & 114.570 & 123.410 & 60.780 & 2.890 & 1.380 & 1.010 & 1.518 & -0.200 & -0.807 & 1.614 & - & - \\
\hline $13 \mathrm{H}-1,107-109$ & 114.870 & 123.690 & 48.790 & 1.274 & 1.376 & 0.713 & 1.316 & -0.808 & -0.826 & 1.395 & - & - \\
\hline $13 \mathrm{H}-1,117-119$ & 114.970 & 123.740 & 66.950 & 2.080 & 1.610 & 1.070 & 1.375 & -0.076 & -0.365 & 1.273 & - & - \\
\hline $13 \mathrm{H}-2,19-21$ & 115.490 & 124.170 & 48.150 & 2.670 & 1.650 & 0.920 & 1.352 & -0.176 & -0.285 & 1.313 & - & - \\
\hline $13 \mathrm{H}-2,35-37$ & 115.650 & 124.320 & 54.060 & 2.585 & 1.856 & 1.638 & 1.106 & -0.841 & -0.620 & 1.408 & - & - \\
\hline $13 \mathrm{H}-2,67-69$ & 115.970 & 124.610 & 59.210 & 4.540 & 0.230 & 6.410 & 1.306 & -0.100 & -0.482 & 1.439 & - & - \\
\hline $13 \mathrm{H}-2,94-96$ & 116.240 & 124.860 & 62.360 & 2.420 & 2.050 & 0.780 & 1.125 & -0.260 & -0.635 & 1.500 & - & - \\
\hline $13 \mathrm{H}-2,106-108$ & 116.360 & 124.970 & 58.020 & 3.293 & 3.977 & 5.249 & 1.549 & -0.586 & -0.152 & 1.500 & - & - \\
\hline $13 \mathrm{H}-3,25-27$ & 117.050 & 125.600 & 63.150 & 3.590 & 1.480 & 0.250 & 1.249 & -0.167 & -0.769 & 1.129 & - & - \\
\hline $13 \mathrm{H}-3,33-35$ & 117.130 & 125.680 & 63.290 & 3.299 & 2.108 & 0.733 & 1.694 & -0.554 & 0.330 & 0.062 & - & - \\
\hline $13 \mathrm{H}-3,69-71$ & 117.490 & 126.010 & 73.810 & 5.790 & 3.030 & 1.380 & 1.611 & -1.216 & -0.527 & 1.244 & - & - \\
\hline $13 \mathrm{H}-3,105-107$ & 117.850 & 126.340 & 67.910 & 6.270 & 4.478 & 1.827 & 1.726 & -1.143 & -0.612 & 1.383 & - & - \\
\hline $13 \mathrm{H}-3,113-115$ & 117.930 & 126.410 & 71.050 & 4.510 & 2.310 & 1.280 & 1.819 & -0.315 & -0.027 & 1.547 & - & - \\
\hline $13 \mathrm{H}-4,25-27$ & 118.500 & 126.950 & 21.310 & 0.150 & 0.150 & 0.450 & - & - & - & - & - & - \\
\hline $13 \mathrm{H}-4,43-45$ & 118.730 & 127.110 & 23.730 & 0.000 & 8.398 & 10.575 & - & - & - & - & - & - \\
\hline $13 \mathrm{H}-4,78-80$ & 119.080 & 127.320 & 87.230 & 0.000 & 0.290 & 0.290 & - & - & - & - & - & - \\
\hline $13 \mathrm{H}-4,109-111$ & 119.390 & 127.500 & 85.710 & 0.080 & 0.170 & 0.080 & - & - & - & - & - & - \\
\hline $13 \mathrm{H}-5,13-15$ & 119.930 & 127.830 & 66.230 & 0.140 & 1.430 & 3.710 & - & - & - & - & -1.549 & -3.040 \\
\hline $13 \mathrm{H}-5,79-81$ & 120.590 & 128.490 & 84.930 & 0.240 & 0.480 & 0.240 & - & - & - & - & -0.929 & -2.593 \\
\hline $13 \mathrm{H}-5,103-105$ & 120.830 & 128.730 & 56.880 & 0.107 & 0.215 & 1.075 & - & - & - & - & - & - \\
\hline $13 \mathrm{H}-6,24-26$ & 121.540 & 129.440 & 48.790 & 1.642 & 3.727 & 2.653 & 0.940 & -2.323 & - & - & -1.800 & -3.638 \\
\hline $13 \mathrm{H}-6,37-39$ & 121.670 & 129.570 & 20.250 & 0.120 & 0.120 & 0.120 & - & - & - & - & - & - \\
\hline $13 \mathrm{H}-6,79-81$ & 122.090 & 129.990 & 26.490 & 0.370 & 0.370 & 7.440 & 1.188 & -1.648 & - & - & - & - \\
\hline $13 \mathrm{H}-6,99-101$ & 122.290 & 130.190 & 70.910 & 0.390 & 3.980 & 0.390 & 0.921 & -1.740 & - & - & -0.499 & -3.221 \\
\hline $13 \mathrm{H}-6,117-119$ & 122.470 & 130.870 & 19.780 & 6.601 & 4.950 & 0.825 & 0.992 & -1.698 & - & - & -2.097 & -3.142 \\
\hline $13 \mathrm{H}-7,12-14$ & 122.920 & 131.320 & 65.930 & 0.203 & 1.464 & 1.993 & - & - & - & - & -0.598 & -3.264 \\
\hline $13 \mathrm{H}-7,29-31$ & 123.090 & 131.490 & 82.590 & 1.760 & 2.460 & 1.580 & - & - & - & - & -0.570 & -3.385 \\
\hline $14 X-1,50-52$ & 123.800 & 137.570 & 88.330 & 13.220 & 5.841 & 1.155 & - & - & - & - & -1.081 & -3.662 \\
\hline $14 X-1,54-57$ & 123.840 & 137.670 & 65.450 & 11.580 & 3.580 & 1.510 & - & - & - & - & -0.832 & -3.931 \\
\hline $14 \mathrm{X}-1,72-75$ & 124.020 & 138.136 & 76.220 & 17.560 & 6.350 & 1.077 & - & - & - & - & - & - \\
\hline $14 \mathrm{X}-1,101-103$ & 124.310 & 138.890 & 92.500 & 38.370 & 3.369 & 1.987 & - & - & - & - & - & - \\
\hline $14 \mathrm{X}-1,119-122$ & 124.490 & 139.249 & 89.610 & 1.280 & 0.560 & 0.210 & - & - & - & - & - & - \\
\hline $14 \mathrm{X}-2,21-23$ & 125.020 & 140.700 & 56.660 & 14.160 & 2.695 & 1.888 & - & - & - & - & -1.966 & -2.416 \\
\hline $14 \mathrm{X}-2,114-116$ & 125.940 & 143.098 & 86.660 & 1.206 & 0.469 & 0.000 & - & - & - & - & -1.331 & -3.350 \\
\hline $14 \mathrm{X}-3,40-42$ & 126.700 & 145.046 & 90.000 & 0.197 & 0.413 & 1.329 & - & - & - & - & - & - \\
\hline $14 \mathrm{X}-3,124-126$ & 127.540 & 147.368 & 80.000 & 0.000 & 0.362 & 0.587 & - & - & - & - & - & - \\
\hline $14 \mathrm{X}-4,14-16$ & 127.940 & 148.370 & 83.330 & 0.609 & 1.160 & 1.044 & - & - & - & - & - & - \\
\hline $14 \mathrm{X}-4,120-123$ & 129.000 & 150.700 & 57.500 & 0.958 & 0.243 & 0.343 & - & - & - & - & - & - \\
\hline $14 X-5,19-21$ & 129.490 & 151.716 & 62.500 & 5.507 & 3.452 & 2.670 & - & - & - & - & - & - \\
\hline $14 X-5,97-100$ & 130.270 & 153.427 & 64.160 & 22.870 & 4.451 & 1.432 & - & - & - & - & - & - \\
\hline
\end{tabular}

crease in abundance toward the bottom of the sequence (from Sample 160-967A-13H-7, 12-14 cm, down to the terminal depth). Several samples from 160-967A-13H-4, 109-111 cm, down to Core 160967A-15X, contain a planktonic foraminiferal assemblage that includes $G$. obliquus, G. quadrilobatus, O. universa, and dominant dwarf forms (T. quinqueloba) associated with brackish water ostracodes. A peak of major abundance of these forms is observed from Sample 160-13H-6, 37-39 cm, to 13H-6, 79-81 cm (Fig. 4; Table 5). The attribution of these sediments to the Messinian is based on (1) the principle of stratigraphic superposition, and (2) the occurrence of typical Messinian brackish ostracodes with intact bivalve which are occasionally recorded from Sample $160-13 \mathrm{H}-4,109-111 \mathrm{~cm}$, to as far down as Section 160-967A-15X-4

\section{Benthic Foraminifers}

Benthic foraminifer assemblages are well preserved and well diversified throughout Zone MP12 and part of Zone MP11 above the Sphaeroidinellopsis acme event. Species diversity varies from 9 to 32 species per sample, with an average of 22 (Table 6). Assemblages include Gyroidinoides laevigatus, Oridorsalis stellatus, Siphonina reticulata, Uvigerina pygmaea, Globocassidulina subglobosa, and Pleurostomella alternans. Minor components include Martinottiella perparva, M. communis, Karreriella bradyi, Valvulineria marmorea, and Brizalina punctata. Deep Atlantic species Parrelloides bradyi and $P$. robertsonianus (Hasegawa et al., 1990; McKenzie et al., 1990; R. Sprovieri, pers. comm., 1996) are observed from the termination of the Sphaeroidinellopsis acme event (Sample 160-967A-13H-2, $106-108 \mathrm{~cm}$ ) upward (Plates 1-2). Species diversity and abundance of benthic foraminifers decrease from Sample 160-967A-13H-3, 25$27 \mathrm{~cm}$, down to $13 \mathrm{H}-3,113-115 \mathrm{~cm}$. The species present in this interval include $O$. stellatus. G. laevigatus, $G$. subglobosa, and $P$. quadriloba. Messinian sediments yield a very poor benthic foraminiferal assemblage, which consists of Ammonia tepida and rarely Elphidium sp. (Table 5; Pl. 3, Figs. 1a-c)

\section{Ostracodes}

Ostracodes are present in Messinian sediments (Pl. 3). Cyprideis pannonica, interpreted and described in previous studies in the Eastern Mediterranean deep-sea record as brackish forms (Benson, 1978; Cita et al., 1978), is observed from Sample 160-967A-13H-4, 109$111 \mathrm{~cm}$, to $15 \mathrm{X}-4,6-8 \mathrm{~cm}$. Paratethyan brackish ostracodes probably belonging to the species Loxoconca diaffarovi (Cita et al., 1980) are observed in Sample 160-967A-13H-4, 109-111 cm. These brackish ostracodes are considered in situ as shown by the occurrence of several specimens with the valves still closed.

\section{Site 969 (Mediterranean Ridge)}

The Mediterranean Ridge drill site was planned to reconstruct the paleoceanographic evolution of the Eastern Mediterranean, and, consequently, multiple holes were drilled. Aboard, only Hole 160-969A was biostratigraphically investigated and sampled. Later, Hole 160- 
Table 2. Abundance of size fractions ( $>151 \mu \mathrm{m}, 40-151 \mu \mathrm{m}$, and $<40 \mu \mathrm{m})$, calcium carbonate content, and stable isotopes of $G$. obliquus and $O$. stellatus plotted vs. mbsf and rmed for Hole 969B.

\begin{tabular}{|c|c|c|c|c|c|c|c|c|c|c|}
\hline \multirow{2}{*}{$\begin{array}{l}\text { Core, section, interval } \\
(\mathrm{cm})\end{array}$} & \multicolumn{2}{|c|}{ Depth } & \multirow{2}{*}{$\begin{array}{l}\mathrm{CaCO}_{3} \\
(\mathrm{wt} \%)\end{array}$} & \multicolumn{3}{|c|}{ Size fraction } & \multicolumn{2}{|c|}{$\begin{array}{c}\text { Planktonic } \\
\text { foraminifers }\end{array}$} & \multicolumn{2}{|c|}{$\begin{array}{c}\text { Benthic } \\
\text { foraminifers }\end{array}$} \\
\hline & (mbsf) & (rmcd) & & $>151 \mu \mathrm{m}$ & $0-151 \mu \mathrm{m}$ & $<40 \mu \mathrm{m}$ & $\delta^{13} \mathrm{C}$ & $\delta^{18} \mathrm{O}$ & $\delta^{13} \mathrm{C}$ & $\delta^{18} \mathrm{O}$ \\
\hline \multicolumn{11}{|l|}{ 160S-969B- } \\
\hline $11 \mathrm{H}-1,31-33$ & 88.720 & 123.857 & 47.110 & 0.240 & 0.130 & 0.050 & - & - & - & - \\
\hline $11 \mathrm{H}-1,100-102$ & 89.410 & 124.508 & 82.230 & 0.007 & 1.460 & 0.950 & 1.694 & -0.671 & -0.771 & 1.219 \\
\hline $11 \mathrm{H}-2,30-32$ & 90.220 & 124.959 & 70.250 & 0.170 & 1.600 & 0.980 & - & - & -0.626 & 1.354 \\
\hline $11 \mathrm{H}-2,100-102$ & 90.910 & 125.614 & 82.230 & 0.040 & 0.170 & 0.060 & - & - & -1.716 & 0.998 \\
\hline $11 \mathrm{H}-3,40-42$ & 91.810 & 125.711 & 69.420 & 0.120 & 0.060 & 0.040 & - & - & - & - \\
\hline $11 \mathrm{H}-3,130-132$ & 92.710 & 126.924 & 84.030 & 0.070 & 1.550 & 0.960 & 1.724 & -0.776 & -0.560 & 0.859 \\
\hline $11 \mathrm{H}-4,40-42$ & 93.310 & 127.268 & 92.440 & 0.340 & 2.790 & 1.350 & 1.580 & -0.434 & -0.680 & 1.424 \\
\hline $11 \mathrm{H}-4,120-122$ & 94.110 & 127.719 & 71.850 & 0.210 & 1.920 & 0.890 & 1.579 & -0.604 & -0.773 & 0.894 \\
\hline $11 \mathrm{H}-5,3-5$ & 94.430 & 127.980 & 75.260 & 15.270 & 6.750 & 0.190 & 1.460 & -1.497 & - & - \\
\hline $11 \mathrm{H}-5,7-9$ & 94.480 & 128.010 & 84.030 & 0.970 & 1.700 & 0.510 & 1.478 & -1.192 & 1.617 & -0.116 \\
\hline $11 \mathrm{H}-5,30-32$ & 94.710 & 128.140 & 89.080 & 0.300 & 2.480 & 1.300 & 1.710 & - & 1.011 & - \\
\hline $11 \mathrm{H}-5,50-52$ & 94.910 & 128.270 & 68.340 & 4.540 & 1.870 & 1.130 & 1.519 & -0.230 & -0.637 & 1.511 \\
\hline $11 \mathrm{H}-5,70-72$ & 95.110 & 128.380 & 73.860 & 0.710 & 2.350 & 1.120 & 1.916 & -0.847 & -0.925 & 0.852 \\
\hline $11 \mathrm{H}-5,80-82$ & 95.210 & 128.450 & 71.120 & 6.230 & 4.590 & 1.250 & 1.282 & -0.914 & 1.299 & 0.948 \\
\hline $11 \mathrm{H}-5,91-92$ & 95.310 & 128.520 & 80.500 & 0.900 & 1.100 & 1.490 & 1.781 & -1.270 & - & - \\
\hline $11 \mathrm{H}-5,117-119$ & 95.580 & 128.730 & 89.630 & 0.730 & 3.120 & 1.250 & 1.976 & -0.549 & -0.979 & 1.018 \\
\hline $11 \mathrm{H}-5,120-122$ & 95.610 & 128.740 & 79.100 & 4.680 & 2.380 & 0.960 & 1.840 & -1.260 & -0.890 & 1.396 \\
\hline $11 \mathrm{H}-5,145-147$ & 95.860 & 129.010 & 83.400 & 1.850 & 5.390 & 2.030 & 1.793 & -1.312 & - & - \\
\hline $11 \mathrm{H}-6,9-10$ & 95.990 & 129.140 & 75.400 & 5.900 & 1.610 & 0.530 & 1.314 & -0.073 & - & - \\
\hline $11 \mathrm{H}-6,19-20$ & 96.090 & 129.240 & 73.780 & 4.140 & 0.460 & 0.460 & 1.324 & -0.265 & - & - \\
\hline $11 \mathrm{H}-6,29-30$ & 96.190 & 129.340 & 67.290 & 7.420 & 4.770 & 1.060 & 1.445 & -0.192 & - & - \\
\hline $11 \mathrm{H}-6,39-40$ & 96.290 & 129.440 & 71.810 & 3.160 & 1.580 & 0.520 & 1.592 & -0.724 & - & - \\
\hline $11 \mathrm{H}-6,49-50$ & 96.390 & 129.540 & 39.720 & 14.660 & 2.820 & 0.560 & 1.592 & -0.834 & - & - \\
\hline $11 \mathrm{H}-6,59-60$ & 96.490 & 129.640 & 81.810 & 10.840 & 4.330 & 2.160 & 1.535 & -1.213 & - & - \\
\hline $11 \mathrm{H}-6,69-70$ & 96.590 & 129.740 & 70.540 & 23.150 & 5.140 & 0.850 & 1.411 & -1.065 & - & - \\
\hline $11 \mathrm{H}-6,79-80$ & 96.690 & 129.840 & 85.130 & 4.520 & 1.690 & 0.560 & 1.689 & -0.390 & - & - \\
\hline $11 \mathrm{H}-6,90-91$ & 96.800 & 129.950 & 72.970 & 4.040 & 0.510 & 0.510 & 1.593 & -0.171 & - & - \\
\hline $11 \mathrm{H}-6,98-99$ & 96.880 & 130.030 & 69.090 & 3.390 & 1.930 & 0.960 & 1.725 & -0.323 & - & - \\
\hline $11 \mathrm{H}-6,108-109$ & 96.960 & 130.130 & 74.540 & 3.630 & 1.810 & 0.450 & 1.630 & -0.988 & - & - \\
\hline $11 \mathrm{H}-6,117-118$ & 97.070 & 130.220 & 74.590 & 7.680 & 5.760 & 1.280 & 1.352 & -1.401 & - & - \\
\hline $11 \mathrm{H}-6,124-126$ & 97.140 & 130.300 & 50.910 & 7.180 & 2.780 & 0.920 & 1.348 & -1.468 & - & - \\
\hline $11 \mathrm{H}-6,128-129$ & 97.180 & 130.330 & 13.780 & 1.510 & 0.000 & 0.750 & - & - & - & - \\
\hline $11 \mathrm{H}-6,139-140$ & 97.290 & 130.440 & 12.970 & 1.380 & 0.690 & 0.690 & 0.857 & -1.681 & - & - \\
\hline $11 \mathrm{H}-7,8-10$ & 97.490 & 130.640 & 16.000 & 0.062 & 0.240 & 2.560 & 1.274 & -1.293 & - & - \\
\hline $11 \mathrm{H}-7,40-42$ & 97.810 & 130.960 & 14.000 & 0.000 & 0.070 & 0.035 & 0.174 & 0.282 & - & - \\
\hline
\end{tabular}

969E was sampled in detail to fulfill the sample request aimed to reconstruct the Miocene/Pliocene boundary sequence. The study was performed, but a clear gap has been documented in Section 160969E-11H-6, $25 \mathrm{~cm}$, at a lithologic change between Pliocene oozes with sapropels and gray barren clay. Consequently, additional sampling was done in Hole 969B, which proved to be the most complete and least disturbed Miocene/Pliocene boundary sequence at Site 969.

\section{Hole $969 B$}

Hole 969B is located on the Mediterranean Ridge ( $33^{\circ} 50.399$ N, $24^{\mathrm{O}} 53.065 \mathrm{E}$, water depth of $2200 \mathrm{~m}$, penetration of $108.3 \mathrm{~m}$ ). Eleven cores were retrieved with the APC from 0 to $97.9 \mathrm{~m}$. Recovery throughout was close to $100 \%$. The sedimentary sequence recovered in this hole consists of Pliocene-Quaternary nannofossil ooze and nannofossil clay, with more than 80 sapropels from the lower Pliocene through the Holocene. Ten sapropels were observed in Core 160-969B-11H where the Miocene/Pliocene boundary was expected $(11 \mathrm{H}-1,20-40 \mathrm{~cm} ; 11 \mathrm{H}-1,115-120 \mathrm{~cm} ; 11 \mathrm{H}-2,40-47 \mathrm{~cm} ; 11 \mathrm{H}-3$, $27-45 \mathrm{~cm} ; 11 \mathrm{H}-3,80-97 \mathrm{~cm} ; 11 \mathrm{H}-5,0-17 \mathrm{~cm} ; 11 \mathrm{H}-5,85-97 \mathrm{~cm}$; $11 \mathrm{H}-5,135-150 \mathrm{~cm} ; 11 \mathrm{H}-6,50-77 \mathrm{~cm}$; and $11 \mathrm{H}-6,120-125 \mathrm{~cm})$. Gray clay was found in Section 160-969B-11H-6, $125 \mathrm{~cm}$, down to the base of Hole 969B (Fig. 3).

\section{Planktonic Foraminifers}

Planktonic foraminiferal assemblages are well preserved and scarce to abundant through the early Pliocene sequence. Zone MP12 is identified in Sample 160-969B-11H-1, 31-33 cm, and $11 \mathrm{H}-1$, 100-102 cm, only. Faunal assemblages include common to abundant Orbulina universa, Globigerinoides obliquus, G. quadrilobatus, G. sacculifer, G. trilobus, Globorotalia margaritae, Globigerina falconensis, Globigerina bulloides, Globigerinita juvenilis, G. glutinata, Neogloboquadrina acostaensis dextral, Zeaglobigerina woodi, Tur- borotalita quinqueloba, Sphaeroidinellopsis spp., Zeaglobigerina decoraperta, Z. nepenthes group, and rare Globigerinella obesa, G. siphonifera, and Globigerinita uvula.

Zone MPI1 is identified from Sample 160-969B-11H-2, 30-32 $\mathrm{cm}$, to $11 \mathrm{H}-6,124-126 \mathrm{~cm}$. Its thickness is $6.92 \mathrm{~m}$. From Sample 160-969B-11H-2, 30-32 cm, to $11 \mathrm{H}-3,130-132 \mathrm{~cm}$, planktonic foraminiferal assemblages are similar to those observed in Hole 967A. The Sphaeroidinellopsis acme event is observed from Sample 160969B-11H-4, 40-42 cm, to $11 \mathrm{H}-6,49-50 \mathrm{~cm}$, with the exception of one sample barren of Sphaeroidinellopsis (Sample 160-969B-11H-6, $39-40 \mathrm{~cm}$ ). Its thickness is $3.08 \mathrm{~m}$. Nine sapropels are recorded in the MPI1 biozone. The Sphaeroidinellopsis group is also very rare to absent from Sample 160-969B-11H-6, 59-60 to $11 \mathrm{H}-6,124-126 \mathrm{~cm}$.

The sediments below Sample 160-969B-11H-6, 124-126 cm consist of gray to dark gray clay. From Sample 160-969B-11H-6, 128$129 \mathrm{~cm}$, downward, the abundances of planktonic foraminifers decreases and the assemblage is dominantly composed of the smallsized $(<125 \mu \mathrm{m})$ species $T$. quinqueloba together with rarer largesized specimens of T. trilobus, G. obliquus, G. bulloides, Z. woodi, N. acostaensis, Sphaeroidinellopsis spp., and O. universa (Table 7).

Although the sediments in this interval contain some marine fauna, they are interpreted to be Messinian "lacustrine deposits" because of the dominant presence of gray clay.

\section{Benthic Foraminifers}

Benthic foraminiferal assemblages are very scarce and show very low diversity throughout. Species abundance varies from 0 to 6 specimens per sample (Table 8). In Zones MP12 and MP11, benthic assemblages fluctuate in abundance from very rare to rare and include Siphonina reticulata, G. subglobosa, O. stellatus, C. italicus, G. laevigatus, and A. helicinus. Abundance and species diversity decrease from Sample 160-969B-11H-5, 120-122 cm, downward. The interval where Sphaeroidinellopsis spp. are absent or rare is barren of 
Table 3. Abundance of size fractions ( $>151 \mu \mathrm{m}, 40-151 \mu \mathrm{m}$, and $<40 \mu \mathrm{m})$, calcium carbonate content, and stable isotopes of $G$. obliquus and $O$. stellatus, plotted vs. rmed for Hole 969E.

\begin{tabular}{|c|c|c|c|c|c|c|c|c|c|}
\hline \multirow[b]{2}{*}{$\begin{array}{l}\text { Core, section, } \\
\text { interval }(\mathrm{cm})\end{array}$} & \multirow[b]{2}{*}{$\begin{array}{l}\text { Depth } \\
\text { (rmcd) }\end{array}$} & \multirow[b]{2}{*}{$\begin{array}{c}\mathrm{CaCO}_{3} \\
(\mathrm{wt} \%)\end{array}$} & & ze fractic & & Planktoni & raminifers & Benthic $\mathrm{f}$ & aminifers \\
\hline & & & $>151 \mu \mathrm{m}$ & $\begin{array}{c}40-151 \\
\mu \mathrm{m}\end{array}$ & $<400 \mu \mathrm{m}$ & $\delta^{13} \mathrm{C}$ & $\delta^{18} \mathrm{O}$ & $\delta^{13} \mathrm{C}$ & $\delta^{18} \mathrm{O}$ \\
\hline $160-969 \mathrm{E}$ & & & & & & & & & \\
\hline $11 \mathrm{H}-1,5-8$ & 117.400 & 63.000 & 1.821 & 3.201 & 1.341 & 1.580 & -0.149 & -0.474 & 1.311 \\
\hline $11 \mathrm{H}-1,14-17$ & 117.453 & 62.000 & 1.594 & 3.405 & 1.280 & 1.544 & -0.158 & -0.418 & 1.405 \\
\hline $11 \mathrm{H}-1,24-27$ & 117.512 & 57.000 & 2.355 & 4.503 & 1.632 & 1.511 & -0.169 & -0.193 & 1.459 \\
\hline $11 \mathrm{H}-1,34-37$ & 117.571 & 52.000 & 1.608 & 3.455 & 1.282 & 1.666 & -0.473 & -0.580 & 1.309 \\
\hline $11 \mathrm{H}-1,46-49$ & 117.642 & 48.000 & 1.794 & 3.362 & 1.471 & 1.652 & -0.336 & -0.589 & 1.255 \\
\hline $11 \mathrm{H}-1,53-56$ & 117.683 & 48.000 & 2.467 & 4.241 & 1.602 & 1.976 & -0.399 & -0.605 & 1.263 \\
\hline $11 \mathrm{H}-1,84-87$ & 117.899 & 56.000 & 1.597 & 3.427 & 1.486 & 1.516 & -0.555 & -0.883 & 1.639 \\
\hline $11 \mathrm{H}-1,95-97$ & 117.983 & 56.000 & 2.600 & 4.369 & 3.079 & 1.482 & -0.609 & -0.916 & 1.609 \\
\hline $11 \mathrm{H}-1,104-107$ & 118.060 & 56.000 & 2.477 & 4.360 & 1.361 & 1.619 & -0.578 & -0.735 & 1.368 \\
\hline $11 \mathrm{H}-1,115-117$ & 118.144 & 63.000 & 3.212 & 4.832 & 1.664 & 1.588 & -0.392 & -0.754 & 1.488 \\
\hline $11 \mathrm{H}-2,5-8$ & 118.470 & 66.000 & 3.333 & 4.386 & 1.678 & 1.480 & -0.204 & -0.533 & 1.393 \\
\hline $11 \mathrm{H}-2,14-17$ & 118.552 & 62.000 & 3.130 & 4.319 & 1.634 & 1.465 & -0.434 & -0.418 & 1.485 \\
\hline $11 \mathrm{H}-2,24-27$ & 118.662 & 63.000 & 3.661 & 4.213 & 1.523 & 1.677 & -0.505 & -0.538 & 1.554 \\
\hline $11 \mathrm{H}-2,34-37$ & 118.772 & 60.000 & 4.163 & 4.843 & 1.748 & 1.451 & -0.135 & -0.633 & 1.474 \\
\hline $11 \mathrm{H}-2,44-47$ & 118.881 & 64.000 & 4.225 & 5.883 & 2.004 & 1.340 & -1.280 & -0.947 & 1.444 \\
\hline $11 \mathrm{H}-2,53-56$ & 118.980 & 67.000 & 5.840 & 8.155 & 3.183 & 1.579 & -0.741 & -0.930 & 1.600 \\
\hline $11 \mathrm{H}-2,64-66$ & 119.095 & 68.000 & 6.080 & 7.242 & 3.272 & 1.461 & -0.846 & -0.881 & 1.574 \\
\hline $11 \mathrm{H}-2,74-76$ & 119.204 & 60.000 & 2.618 & 3.320 & 1.176 & 1.710 & -0.432 & -0.480 & 1.567 \\
\hline $11 \mathrm{H}-2,87-89$ & 119.331 & 68.000 & 2.710 & 3.950 & 1.520 & 1.578 & -0.299 & -0.498 & 1.456 \\
\hline $11 \mathrm{H}-2,104-107$ & 119.501 & 66.000 & 5.617 & 4.782 & 2.088 & 1.341 & -0.464 & -0.689 & 1.415 \\
\hline $11 \mathrm{H}-2,114-116$ & 119.594 & 66.000 & 6.598 & 5.007 & 1.994 & 0.626 & -1.881 & -0.587 & 1.484 \\
\hline $11 \mathrm{H}-2,124-127$ & 119.696 & 68.000 & 7.431 & 5.953 & 2.370 & 0.736 & -2.295 & -0.776 & 1.503 \\
\hline $11 \mathrm{H}-2,134-136$ & 119.789 & 70.000 & 6.350 & 6.932 & 2.823 & 0.357 & -2.431 & -0.844 & 1.505 \\
\hline $11 \mathrm{H}-2,144-147$ & 119.891 & 68.000 & 5.806 & 6.652 & 2.976 & 1.252 & -0.394 & -1.088 & 1.555 \\
\hline $11 \mathrm{H}-3,5-8$ & 119.998 & 77.000 & 7.262 & 8.059 & 3.385 & 1.257 & -0.205 & -1.215 & 1.407 \\
\hline $11 \mathrm{H}-3,14-17$ & 120.086 & 78.000 & 6.043 & 6.970 & 2.325 & 1.384 & -0.310 & -1.022 & 1.503 \\
\hline $11 \mathrm{H}-3,24-26$ & 120.174 & 67.000 & 2.484 & 3.461 & 1.045 & 1.628 & -0.195 & -0.401 & 1.729 \\
\hline $11 \mathrm{H}-3,34-37$ & 120.281 & 70.000 & 1.960 & 3.320 & 1.106 & 1.633 & -0.295 & -0.393 & 1.671 \\
\hline $11 \mathrm{H}-3,44-47$ & 120.379 & 80.000 & 2.800 & 4.060 & 1.250 & 1.665 & -0.337 & -0.613 & 1.617 \\
\hline $11 \mathrm{H}-3,57-60$ & 120.505 & 76.000 & 2.810 & 4.557 & 1.531 & 1.753 & -0.426 & -0.523 & 1.538 \\
\hline $11 \mathrm{H}-3,64-66$ & 120.569 & 60.000 & 1.926 & 2.830 & 1.242 & 1.014 & 0.061 & -0.966 & 1.700 \\
\hline $11 \mathrm{H}-3,73-76$ & 120.661 & 66.000 & 2.091 & 2.901 & 1.204 & 1.111 & 0.323 & -0.831 & 1.777 \\
\hline $11 \mathrm{H}-3,87-89$ & 126.087 & 64.000 & 1.491 & 2.446 & 0.585 & 1.325 & -0.083 & -0.790 & 1.916 \\
\hline $11 \mathrm{H}-3,97-100$ & 126.156 & 56.000 & 1.562 & 2.649 & 1.096 & 1.093 & 0.254 & -0.677 & 1.627 \\
\hline $11 \mathrm{H}-3,104-107$ & 126.202 & 55.000 & 1.773 & 2.900 & 0.978 & 1.127 & -0.011 & -0.737 & 1.413 \\
\hline $11 \mathrm{H}-3,114-116$ & 126.265 & 51.000 & 1.858 & 2.590 & 0.985 & 1.181 & -0.015 & -0.623 & 1.702 \\
\hline $11 \mathrm{H}-3,124-127$ & 126.334 & 56.000 & 1.484 & 2.695 & 0.944 & 1.295 & -0.137 & -0.946 & 1.486 \\
\hline $11 \mathrm{H}-3,134-136$ & 126.396 & 64.000 & 0.765 & 1.410 & 0.758 & 1.405 & 0.100 & -0.896 & 1.541 \\
\hline $11 \mathrm{H}-3,144-147$ & 126.465 & 64.000 & 0.037 & 0.130 & 1.184 & 1.459 & -0.522 & -0.847 & 1.228 \\
\hline $11 \mathrm{H}-4,17-20$ & 126.626 & 60.000 & 2.987 & 4.700 & 1.829 & 1.134 & -0.565 & -0.699 & 1.297 \\
\hline $11 \mathrm{H}-4,34-37$ & 126.760 & 67.000 & 0.931 & 2.409 & 0.886 & 1.133 & -0.221 & -0.651 & 1.250 \\
\hline $11 \mathrm{H}-4,44-47$ & 126.840 & 62.000 & 2.584 & 3.230 & 1.233 & 1.299 & -0.036 & -0.617 & 0.980 \\
\hline $11 \mathrm{H}-4,57-60$ & 126.943 & 60.000 & 1.765 & 3.174 & 1.164 & 1.137 & -0.234 & -0.635 & 1.279 \\
\hline $11 \mathrm{H}-4,64-66$ & 126.994 & 62.000 & 3.117 & 3.750 & 1.292 & 0.973 & -0.297 & -0.709 & 1.267 \\
\hline $11 \mathrm{H}-4,74-77$ & 127.078 & 64.000 & 2.902 & 2.909 & 1.219 & 1.249 & -0.142 & -0.888 & 1.175 \\
\hline $11 \mathrm{H}-4,84-87$ & 127.157 & 60.000 & 3.517 & 3.771 & 1.421 & 1.085 & -0.185 & -0.604 & 1.277 \\
\hline $11 \mathrm{H}-4,94-97$ & 127.236 & 66.000 & 4.826 & 6.542 & 2.923 & 1.124 & -0.677 & - & - \\
\hline $11 \mathrm{H}-4,102-104$ & 127.296 & 72.000 & 4.640 & 6.224 & 1.896 & 1.394 & -0.033 & -1.110 & 0.924 \\
\hline $11 \mathrm{H}-4,114-116$ & 127.391 & 66.000 & 3.177 & 3.319 & 1.214 & 1.281 & -0.031 & -0.702 & 1.412 \\
\hline $11 \mathrm{H}-4,124-127$ & 127.474 & 64.000 & 3.349 & 3.285 & 1.133 & 1.202 & -0.005 & -0.472 & 1.462 \\
\hline $11 \mathrm{H}-4,137-139$ & 127.565 & 64.000 & 3.131 & 3.640 & 1.267 & 1.227 & 0.095 & -0.840 & 1.400 \\
\hline $11 \mathrm{H}-4,144-147$ & 127.633 & 61.000 & 2.611 & 3.443 & 1.072 & 1.222 & -0.013 & -0.842 & 1.041 \\
\hline $11 \mathrm{H}-5,4-7$ & 127.712 & 60.000 & 2.716 & 3.452 & 1.129 & 1.279 & -0.189 & -0.600 & 1.211 \\
\hline $11 \mathrm{H}-5,14-17$ & 127.791 & 63.000 & 2.529 & 3.295 & 0.337 & 1.489 & -0.465 & -0.893 & 0.876 \\
\hline $11 \mathrm{H}-5,44-47$ & 128.007 & 74.000 & 2.379 & 3.917 & 2.123 & 1.423 & -0.114 & -0.858 & 0.970 \\
\hline $11 \mathrm{H}-5,54-57$ & 128.062 & 78.000 & 4.087 & 5.380 & 1.785 & 1.391 & -0.230 & -0.939 & 1.078 \\
\hline $11 \mathrm{H}-5,64-66$ & 128.115 & 77.000 & 3.202 & 4.061 & 1.306 & 1.394 & -0.247 & -0.797 & 1.187 \\
\hline $11 \mathrm{H}-5,74-77$ & 128.173 & 70.000 & 4.971 & 4.513 & 1.887 & 1.496 & 0.077 & -0.788 & 1.109 \\
\hline $11 \mathrm{H}-5,84-87$ & 128.229 & 66.000 & 3.008 & 3.018 & 1.282 & 1.485 & -0.099 & -0.828 & 0.954 \\
\hline $11 \mathrm{H}-5,94-97$ & 128.285 & 62.000 & 2.819 & 2.552 & 1.239 & 1.331 & -0.108 & -0.861 & 1.246 \\
\hline $11 \mathrm{H}-5,104-106$ & 128.337 & 60.000 & 1.438 & 2.013 & 1.105 & 1.260 & -0.216 & -0.931 & 1.190 \\
\hline $11 \mathrm{H}-5,111-113$ & 128.376 & 66.000 & 1.377 & 1.797 & 0.981 & 1.669 & -0.636 & -0.591 & 1.187 \\
\hline $11 \mathrm{H}-5,134-137$ & 128.553 & 70.000 & 3.666 & 4.293 & 1.344 & 1.609 & -0.663 & -0.732 & 0.885 \\
\hline $11 \mathrm{H}-5,144-147$ & 128.657 & 64.000 & 2.811 & 3.054 & 1.199 & 1.491 & -0.481 & - & - \\
\hline $11 \mathrm{H}-6,4-7$ & 128.762 & 66.000 & 2.218 & 3.373 & 1.046 & 1.348 & -0.541 & -1.068 & 1.550 \\
\hline $11 \mathrm{H}-6,14-17$ & 128.866 & 87.000 & 1.343 & 2.527 & 1.319 & - & - & - & - \\
\hline $11 \mathrm{H}-6,24-27$ & 130.683 & 18.000 & 0.011 & 0.011 & 0.036 & - & - & - & - \\
\hline $11 \mathrm{H}-6,34-37$ & 130.875 & 14.000 & 0.008 & 0.023 & 0.039 & - & - & - & - \\
\hline $11 \mathrm{H}-6,44-47$ & 131.067 & 14.000 & 0.008 & 0.015 & 0.031 & - & - & - & - \\
\hline $11 \mathrm{H}-6,48-51$ & 131.135 & 14.000 & 0.006 & 0.013 & 0.032 & - & - & - & - \\
\hline $11 \mathrm{H}-6,54-57$ & 131.200 & 16.000 & 0.013 & 0.013 & 0.039 & - & - & - & - \\
\hline $11 \mathrm{H}-6,74-77$ & 131.400 & 14.000 & 0.010 & 0.029 & 0.048 & - & - & - & - \\
\hline $11 \mathrm{H}-6,84-87$ & 131.500 & 12.000 & 0.006 & 0.026 & 0.040 & - & - & - & - \\
\hline $11 \mathrm{H}-6,94-97$ & 131.600 & 13.000 & 0.015 & 0.015 & 0.039 & - & - & - & - \\
\hline $11 \mathrm{H}-6,105-108$ & 131.710 & 14.000 & 0.032 & 0.032 & 0.024 & - & - & - & - \\
\hline $11 \mathrm{H}-6,113-116$ & 131.790 & 13.000 & 0.007 & 0.020 & 0.039 & - & - & - & - \\
\hline $11 \mathrm{H}-6,124-127$ & 131.900 & 16.000 & 0.005 & 0.021 & 0.032 & - & - & - & - \\
\hline $11 \mathrm{H}-6,134-137$ & 132.000 & 16.000 & 0.008 & 0.015 & 0.038 & - & - & - & - \\
\hline $11 \mathrm{H}-6,143-145$ & 132.085 & 14.000 & 0.000 & 0.008 & 0.044 & - & - & - & - \\
\hline
\end{tabular}


Table 4. Distribution and abundance of Pliocene planktonic foraminifers in Hole 967A.

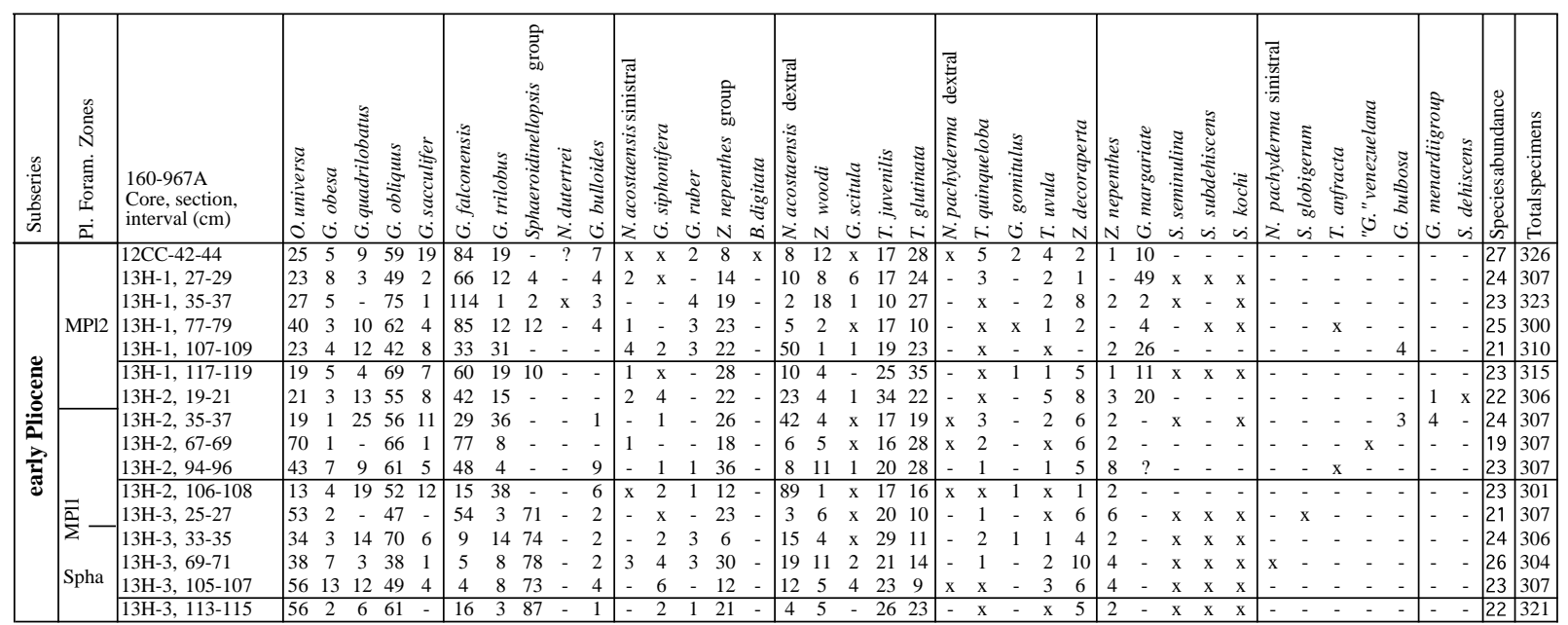

Note: $\mathrm{x}=$ simple occurrence, but not represented in the 300 specimens counted.

Table 5. Distribution of planktonic foraminifers in Messinian and early Pliocene (Zanclean) sediments.

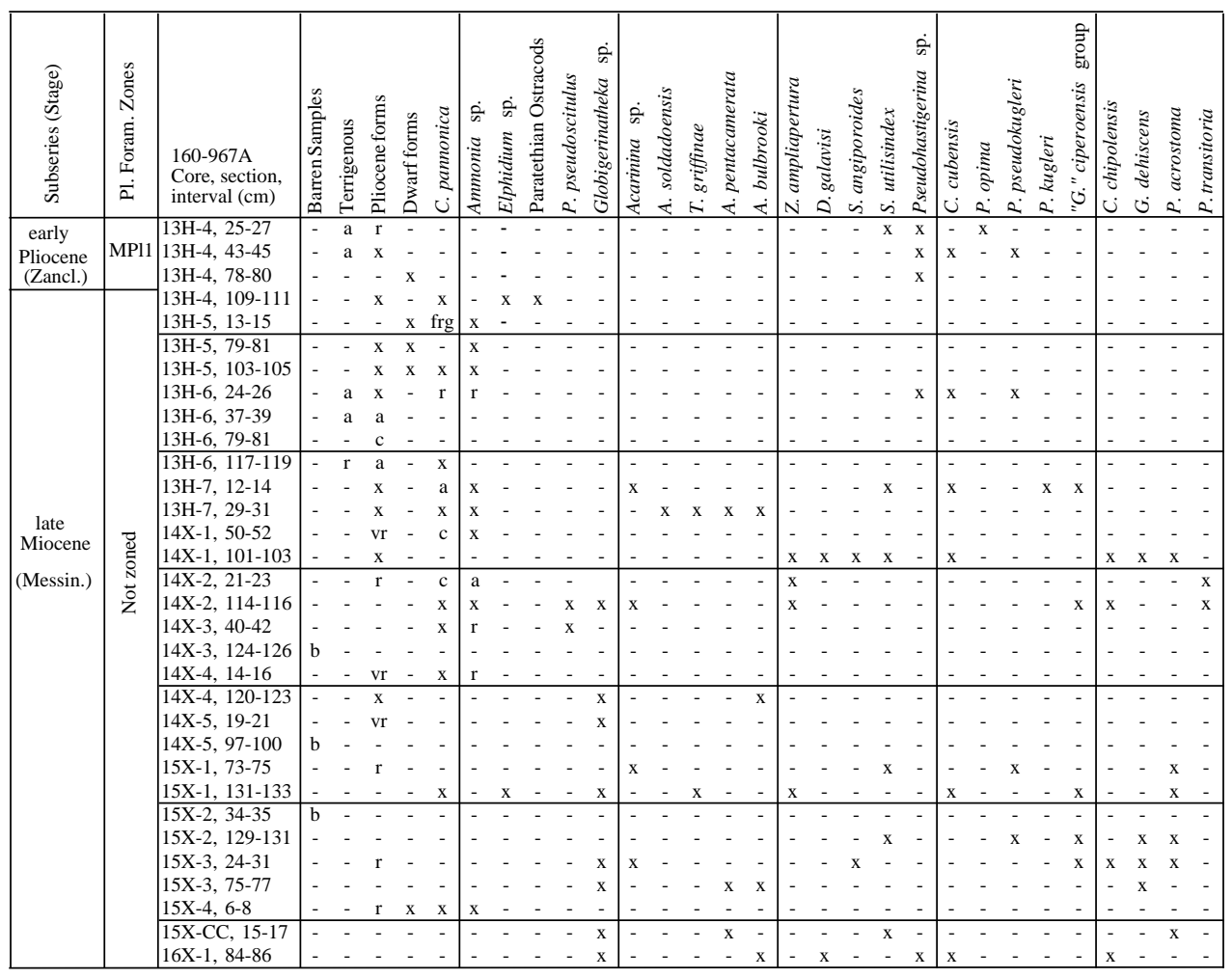

Note: $\mathrm{vr}=$ very rare, $\mathrm{r}=$ rare, $\mathrm{c}=$ common, $\mathrm{a}=$ abundant, $\mathrm{d}=$ dominant, $\mathrm{frg}=$ fragments.

benthic foraminifers (Sample 160-969B-11H-6, 59-60 cm, to $11 \mathrm{H}-6$, $124-126 \mathrm{~cm})$.

\section{Hole $969 E$}

Hole $969 \mathrm{E}$ is located on the Mediterranean Ridge $\left(33^{\circ} 50.462 \mathrm{~N}\right.$, $24^{\mathrm{O}} 52.98 \mathrm{E}$, water depth of 2201.1 , penetration of $\left.97.9 \mathrm{~m}\right)$. Eleven cores were retrieved with the APC from 0 to $97.9 \mathrm{~m}$. Recovery throughout was close to $100 \%$. Coring was terminated when the sed- imentary sequence became extremely firm while drilling for Core $160-969 \mathrm{E}-12 \mathrm{H}$. The sedimentary sequence recovered in this hole is very similar to that recovered at Hole 969B.

Six sapropels were observed in Core $160-969 \mathrm{E}-11 \mathrm{H}$ where the Miocene/Pliocene boundary was expected $(11 \mathrm{H}-1,67-77 \mathrm{~cm} ; 11 \mathrm{H}-$ 3,137 to $11 \mathrm{H}-4,10 \mathrm{~cm} ; 11 \mathrm{H}-4,25-27 \mathrm{~cm} ; 11 \mathrm{H}-5,25-40 \mathrm{~cm} ; 11 \mathrm{H}-$ $5,127-130 \mathrm{~cm}$; and $11 \mathrm{H}-6,15-20 \mathrm{~cm})$. Gray clay was encountered at the top of Section 160-969E-11H-6, $23 \mathrm{~cm}$, down to the end of the hole. Shore-based studies revealed that several faults are present in 
ODP Leg 160 - Hole 967A

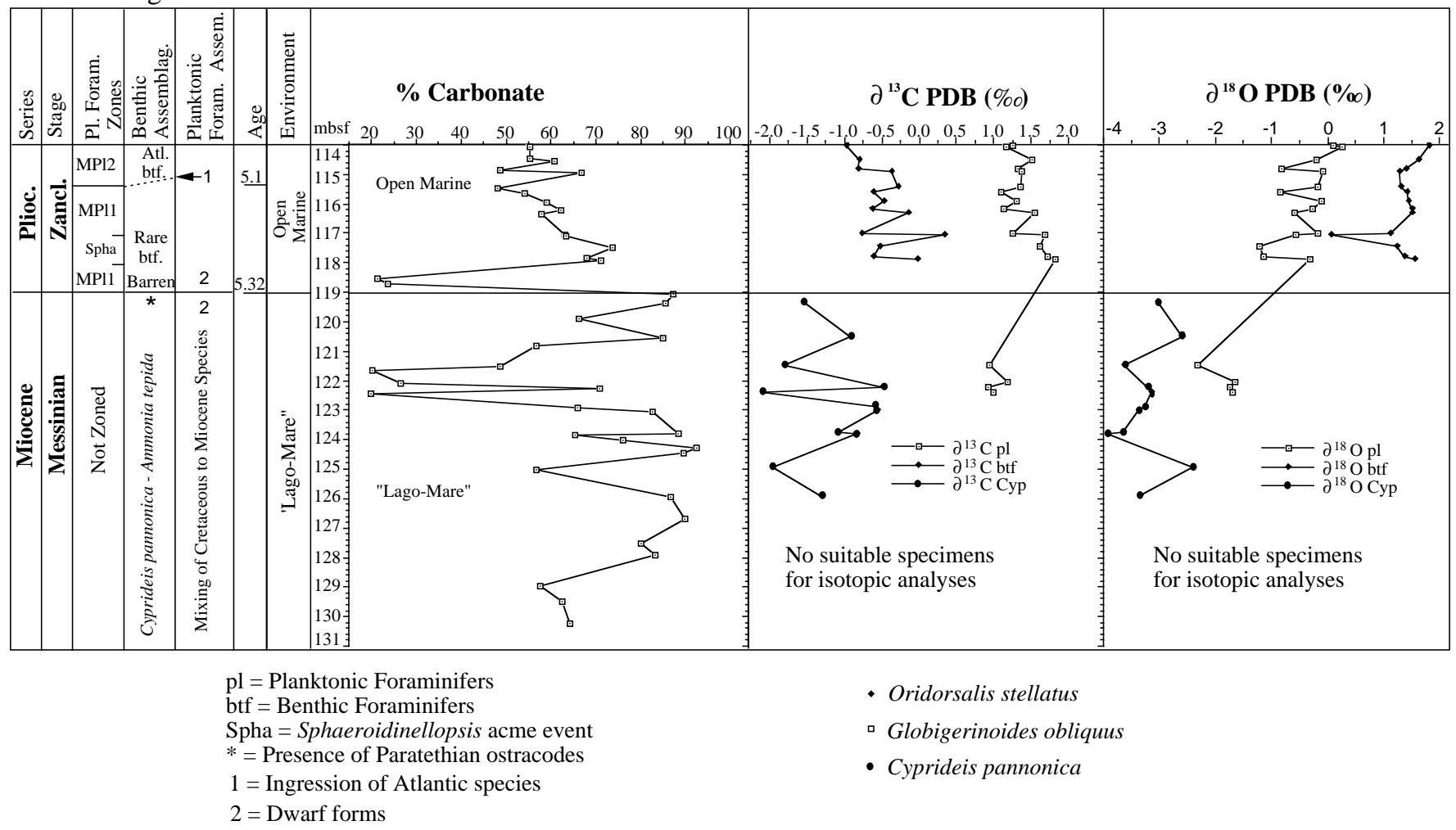

Figure 4. Summary of chemo- and biostratigraphy of the uppermost Miocene and lowermost Pliocene sediments of Hole 967A. Numerical age from Berggren et al. (1995).

this hole. The most important ones occur within Section $11 \mathrm{H}-3$ and at the contact between the calcareous oozes and the gray clay (Sakamoto et al., Chap. 4, this volume; T. Sakamoto, pers. comm., 1997).

\section{Planktonic Foraminifers}

Because a fault was present, Zone MP11 is missing at Hole 969E. Core $160-969 \mathrm{E}-11 \mathrm{H}$ contains sediments attributed to Zone MPl2. Planktonic foraminiferal assemblages are well preserved and rich throughout. They consist of the typical lower Pliocene fauna previously described for Holes 967A and 969B. No range charts were produced for this hole, since the lowermost part of the Pliocene succession is missing.

\section{Benthic Foraminifers}

Benthic foraminifers assemblages from this hole are abundant and rich and include all the species observed for Hole 967A (Table 6).

\section{GEOCHEMICAL RESULTS \\ Calcium Carbonate}

The calcium carbonate content varies from $19 \%$ to more than $80 \%$ of the pelagic oozes in Hole 967A, where the larger amplitude fluctuations are observed. In particular, low calcium carbonate content peaks are observed for Samples 160-967A-13H-4, 25-27 cm and $13 \mathrm{H}-4,43-45 \mathrm{~cm}(21.31 \%$ and $23.75 \%)$ and for Samples 160-967A$13 \mathrm{H}-6,37-39 \mathrm{~cm}, 13 \mathrm{H}-6,79-81 \mathrm{~cm}$, and $13 \mathrm{H}-6,117-119 \mathrm{~cm}$ $(20.25 \%, 26.49 \%$ and $29.78 \%$, respectively; Table 1, Fig. 4). A sharp drop in carbonate content from about $51 \%$ to $13 \%$ and from $87 \%$ to $18 \%$ (Samples 160-969B-11H-6, 128-129 cm, and 160-969E-11H-6,
24-27 cm, respectively) is observed in both Holes 969B and 969E in correspondence to the change in lithology (Tables 1-3; Figs. 4-6).

\section{Stable Isotopes}

The stable isotope stratigraphy for Holes 967A, 969B, and 969E are shown in Figures 4-6, respectively. In Hole 967A, the earliest Pliocene $\delta^{18} \mathrm{O}$ values for both planktonic and benthic foraminifers tend to show an upward enrichment in ${ }^{18} \mathrm{O}$, whereas the $\delta^{13} \mathrm{C}$ values tend toward a depletion in ${ }^{13} \mathrm{C}$ (Fig. 4). Fluctuations in the $\delta^{18} \mathrm{O}$ appear to be related to the fluctuations in abundance of the warm water Globigerinoides group (Fig. 7). There is a notable negative reversal in the $\delta^{13} \mathrm{C}$ values of the benthic foraminifers at the top of the Sphaeroidinellopsis acme event. A similar reversal was observed in a high-resolution study of the earliest Pliocene sediments deposited at ODP Leg 107, Site 652 in the Tyrrhenian Sea (McKenzie et al., 1990). The ostracode $C$. pannonica displays stable isotope values markedly more negative than those observed for either the benthic or planktonic foraminifers (Fig. 4; Table 1).

In Hole 969B, the $\delta^{13} \mathrm{C}$ values appear to be rather invariant for planktonic foraminifers (Fig. 5), whereas fluctuations observed in $\delta^{18} \mathrm{O}$ appear to correspond to fluctuations in the abundance of the warm water Globigerinoides group (Fig. 8). Benthic foraminifers show two major positive shifts in their $\delta^{13} \mathrm{C}$ values in the middle-upper part of the Sphaeroidinellopsis acme event. The uppermost shift coincides with a corresponding but negative excursion in the benthic $\delta^{18} \mathrm{O}$ value. A similar trend was also observed in the Hole 967A sequence and the Tyrrhenian Sea (McKenzie et al., 1990).

In the case of Hole 969E, the isotope stratigraphy tends to be rather monotonous but typical for a marine system. In the upper part of 
Table 6. Distribution and species abundance of Pliocene benthic foraminifers in Hole 967A.

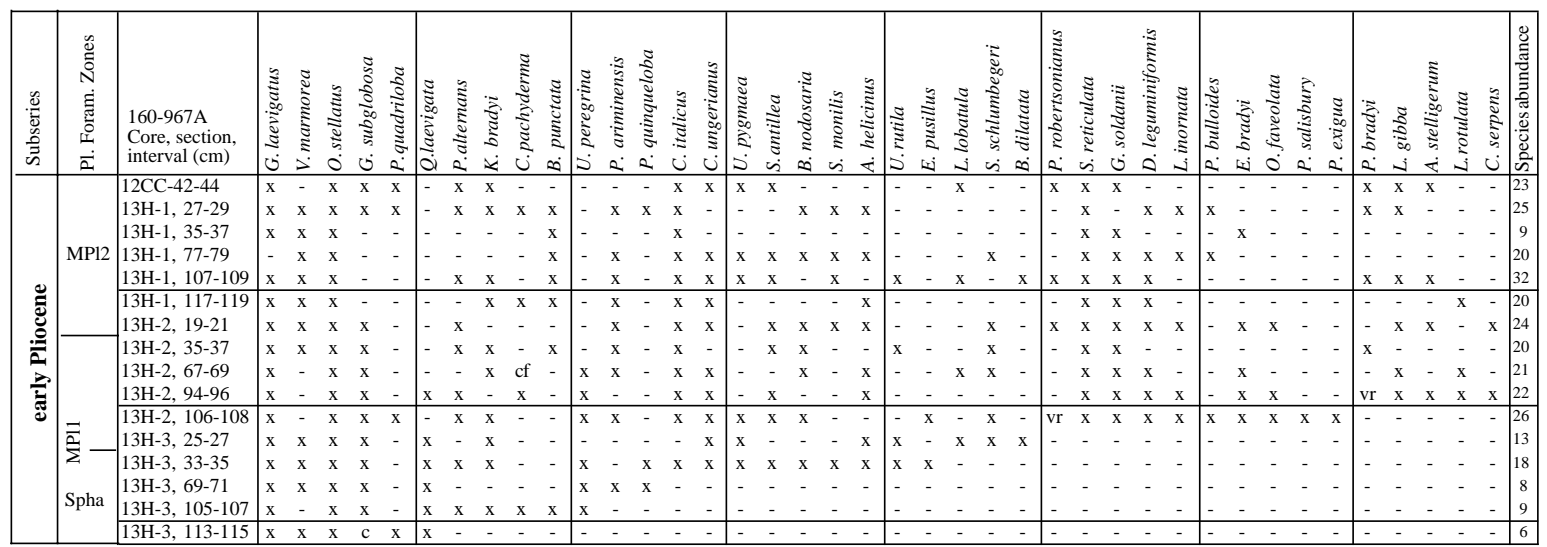

Note: $\mathrm{vr}=$ very rare

Table 6 (continued).

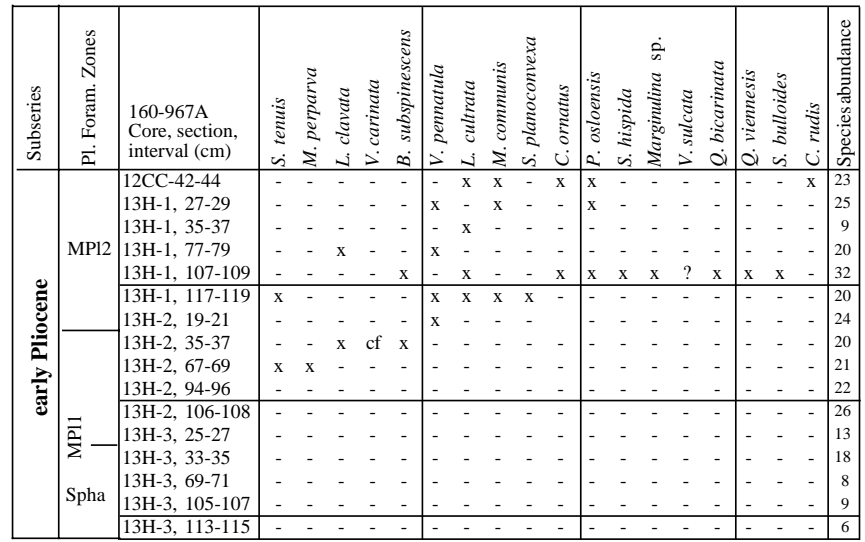

the MP12 Biozone, there is, however, an excursion toward significantly more negative $\delta^{18} \mathrm{O}$ values (Fig. 6 ).

The stable isotope signals of the planktonic foraminifers that occur associated with brackish water ostracodes in Hole 967A are slightly more enriched in the heavier isotopes relative to the ostracodes but are depleted relative to the values for the foraminifers from the overlying marine sequence (Table 1; Fig. 4).

\section{DISCUSSION}

At Hole 967A, located on the base of the northern slope of the Eratosthenes Seamount, the identification of the Miocene/Pliocene boundary coincides with the lower boundary of the lithostratigraphic Unit II and is located between 119.1 and 119.4 mbsf (between Samples 160-967A-13H4, 78-80 cm, and 13H-4, 109-111 cm; Emeis, Robertson, Richter, et al., 1996). At the same depth, a shift from a high content of inorganic and non-marine calcite to a high content of a biogenic calcite typical of the marine pelagic ooze is also observed (Emeis, Robertson, Richter, et al., 1996). The presence of the wellpreserved ostracodes with closed valves in Sample 160-967A-13H-4, 109-111 cm, supports this placement of the Miocene/Pliocene boundary, as it represents the uppermost occurrence of these brackish water species.

At Site 969 , some $700 \mathrm{~km}$ to the west of the previous location, on the inner plateau of the Mediterranean Ridge south of Crete, the identification of the Miocene/Pliocene boundary coincides with a marked lithologic change, which is recorded in three discrete holes (Holes 969A, 969B, 969E), as shown in Figure 9. The overlying unit is represented by early Zanclean nannofossil-foraminiferal oozes with intercalated sapropels, whereas the underlying unit is gray mud, with rarer and generally dwarf microfossils consisting of dominant $T$. quinqueloba. The biostratigraphic correlation shown in Figure 9 demonstrates that the sapropels recorded in Section 160-969E-11H6 are not correlatable with most of sapropels of Core 160-969B-11H, but are younger. A sediment thickness of some $6 \mathrm{~m}$ is missing in Hole 969E. The sedimentary sequences record an unstable tectonic setting during their deposition and immediately afterward. Unfortunately, the penetration was interrupted, and no older Messinian lithologies were reached.

At Hole 969B the Miocene/Pliocene boundary is located between 97.1 and 97.2 mbsf (between Samples 160-969B-11H-6, 124-126 $\mathrm{cm}$, and $11 \mathrm{H}-6,128-129 \mathrm{~cm})$. The pre-Pliocene sediments recovered on the Mediterranean Ridge are fine grained and similar to those recovered in deep-sea settings at DSDP Site 374 on the Messina abyssal plain in the Ionian Basin (Hsü, Montadert, et al., 1978) and Aphrodite Crater on the Western Mediterranean Ridge (Blechschmidt et al., 1982).

The presence of planktonic foraminifers associated with brackish water ostracodes in Hole 967A and with the gray clay in Hole 969B below the interpreted Miocene/Pliocene boundary deserves comment. In fact, the stable isotope composition of $C$. pannonica indicates that this crustacean lived in a Lago Mare environment characterized by brackish water, distinctly different form the marine envi- 
Table 7. Distribution and abundance of Pliocene and upper Messinian planktonic foraminifers in Hole 969B.

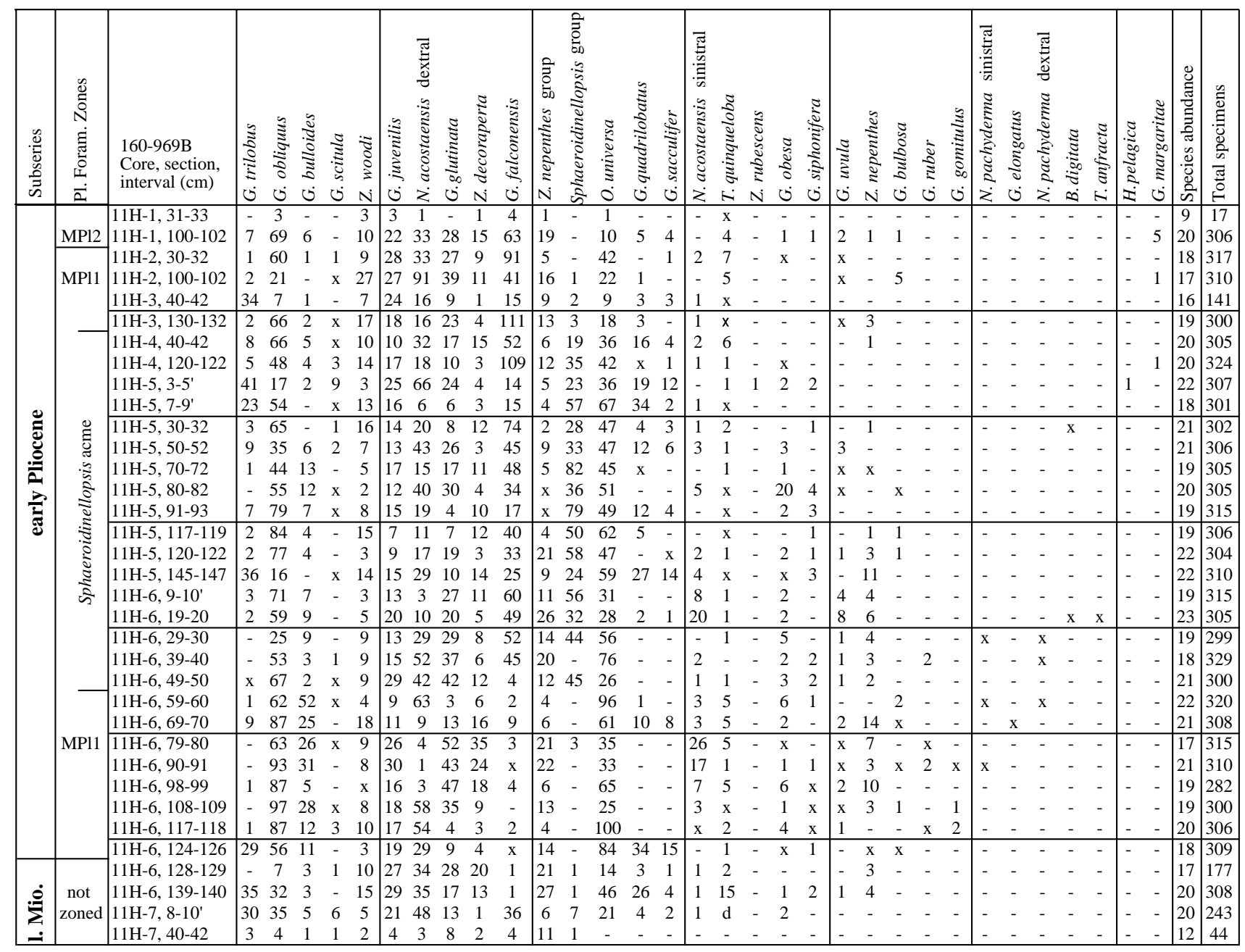

Note: $\mathrm{x}=$ simple occurrence, but not represented in the 300 specimens counted.

ronment. The concurrence of planktonic foraminifers and ostracodes in the same samples representing the uppermost Messinian Lago Mare facies is problematic and indicates either reworking, contamination or temporary incursion of "Atlantic" water into a fully Lago Mare environment.

The isotopic composition of the concurring ostracodes and planktonic foraminifers at Hole 967A reflects a shared environment for at least a short interval, as also pointed out by Cita (1973a). In addition, the interval where the higher abundance of planktonic foraminifers associated with ostracodes is observed coincides with the low calcium carbonate peak between 121.6 and $122.4 \mathrm{~m}$ (Table 1; Fig. 4). Based on these observations, a temporary incursion of "Atlantic" water into a fully Lago Mare environment is the more reasonable explanation for the presence of planktonic foraminifers in sediment deposited before the Zanclean transgression.

The Zanclean transgression appears as a deep-sea flood extending to the distal part of the Eastern Mediterranean. The sedimentary expression of this event differs at the various sites, indicating a variety of situations that influenced the flood, which appears as a rapid rise of sea level with open marine conditions invading previously isolated and depressed ponds. The existence of sills results in the development of cyclically repeated anoxic conditions, as recorded in the Mediterranean drill holes. In other distal locations, however, oxygenation is good, as observed on the Eratosthenes Seamount and in the
Pissouri section measured on western Cyprus (Orszag-Sperber and Rouchy, 1979; Cita et al., unpubl. data).

Repopulation of the Eastern Mediterranean by benthic biota (foraminifers) was gradual. Deep Atlantic taxa (Parrelloides bradyi and $P$. robertsonianus) appeared in correspondence with the termination of the Sphaeroidinellopsis acme event, as also reported by Wright (1980) and McKenzie et al. (1990). The occurrence of a negative reversal in the oxygen isotopic composition of the benthic foraminifers at the end of the Sphaeroidinellopsis acme event may represent a paleoceanographic reversal in the recovery of the Mediterranean to full marine conditions after the terminal flooding. A similar isotopic excursion in the Tyrrhenian Sea was interpreted as the result of a reorganization of the paleocirculation pattern in the Western Mediterranean with the initial opening of a new shallow connection to the Atlantic Ocean, which would become the site of the modern Straits of Gibraltar with continued headward erosion (McKenzie et al., 1990).

Peaks in the abundance of planktonic foraminiferal fauna observed in the Eastern Mediterranean drill sites recall those observed by Sprovieri $(1992,1993)$ in the central Mediterranean, which were interpreted as fluctuations forced by the precession astronomical cycles. Finally, the presence of Atlantic planktonic foraminiferal taxa, such as specimens belonging to the Globorotalia menardii group, at the boundary between the Zones MPI1 and MPI2, indicates that an efficient connection between the Atlantic Ocean and the Eastern Med- 
Table 8. Distribution and species abundance of benthic foraminifers in Hole 969B.

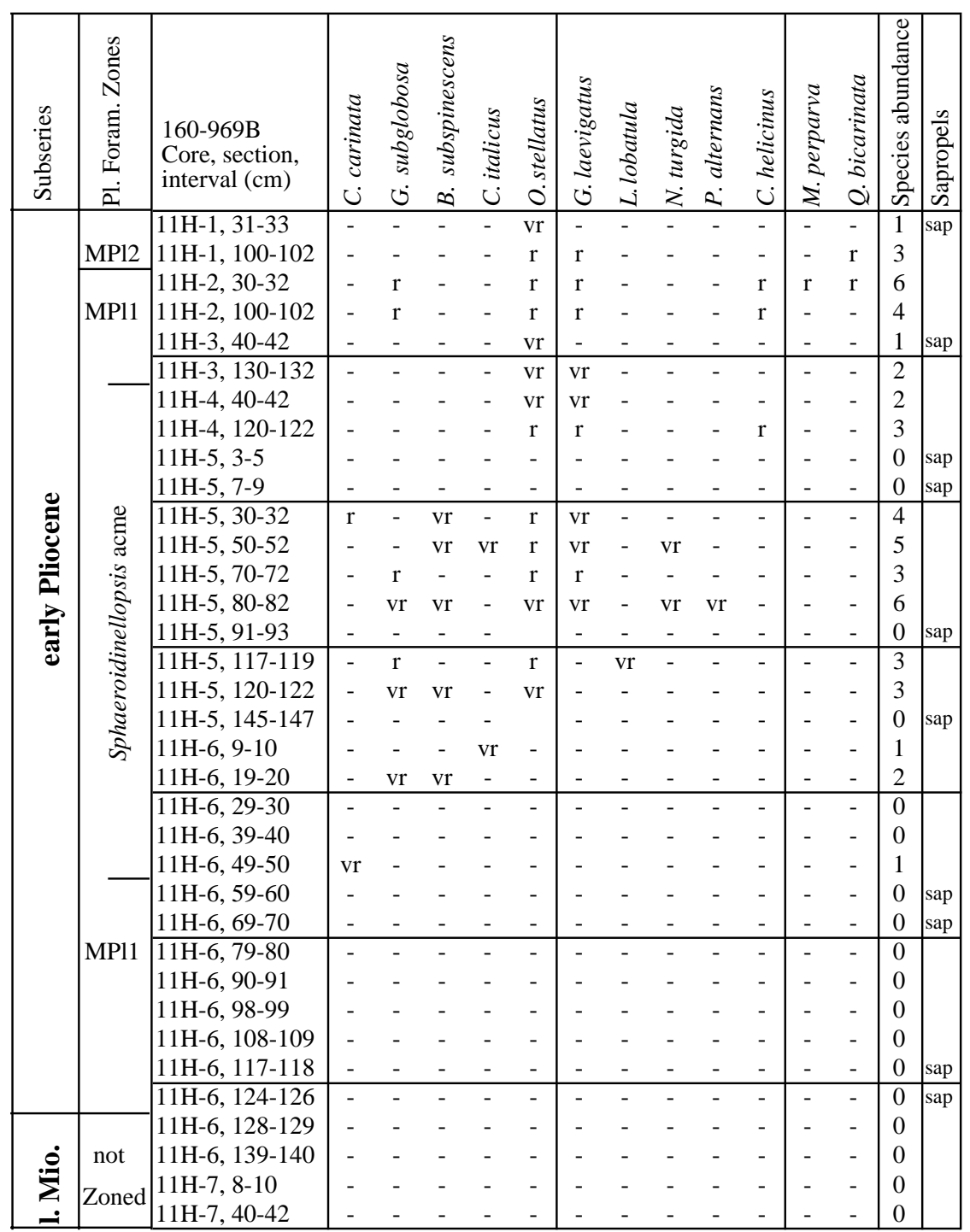

Note: $\mathrm{vr}=$ very rare, $\mathrm{r}=$ rare

iterranean Sea was re-established in this interval, as was previously determined from studies of a continuous Miocene/Pliocene boundary section from ODP Leg 107, Site 652 in the Tyrrhenian Sea (McKenzie et al., 1990).

Several lines of evidence suggest that, in late Messinian and early Zanclean times, the paleobathymetry of the Mediterranean Ridge drill site was deeper than that of the Eratosthenes Seamount drill site. First, the shallow-water, brackish Lago Mare biofacies is present in the more eastern location but was not recorded on the Mediterranean Ridge. Second, the lower Zanclean sediments on the Mediterranean Ridge are much poorer in benthic foraminifers and contain sapropels, whereas those on the Eratosthenes Seamount are well oxygenated and richer in bottom biota.

\section{CONCLUSIONS}

The Miocene/Pliocene boundary (Messinian/Zanclean boundary) as recorded in the ODP Leg 160 drill sites is probably coeval with that defined in Sicily at the type locality, where it has been carefully calibrated. It falls within the Gilbert Chron, five astronomical (precession) cycles in the magnetic reversal below the Thvera event, at the base of the foraminiferal Zone MPl1, with a numerical age of 5.32 $\mathrm{Ma}$, using the time scale of Berggren et al. (1995).

The occurrence of Lago-Mare biofacies at the eastern end-member of the Mediterranean transect south of Cyprus and the strongly negative isotopic signature of the ostracode shells confirm the nonmarine nature of the latest Messinian sediments laid down after the evaporitic episodes, as previously pointed out by Cita et al. (1978) and McKenzie and Ricchiuto (1978). In the Eastern Mediterranean, the deep-sea nature of the earliest Zanclean sediments is also confirmed, as is the progressive repopulation by the benthic communities with deep bathyal Atlantic taxa first appearing 200,000 years after the initial flooding (Wright, 1980; McKenzie et al., 1990). A fully open deep-water connection to the Atlantic Ocean was established coincident with the MP11/MP12 boundary in both the Eastern and Western Mediterranean. In conclusion, from the Leg 160 data set depicting the event stratigraphy across the Miocene/Pliocene boundary 
ODP Leg 160 - Hole 969B

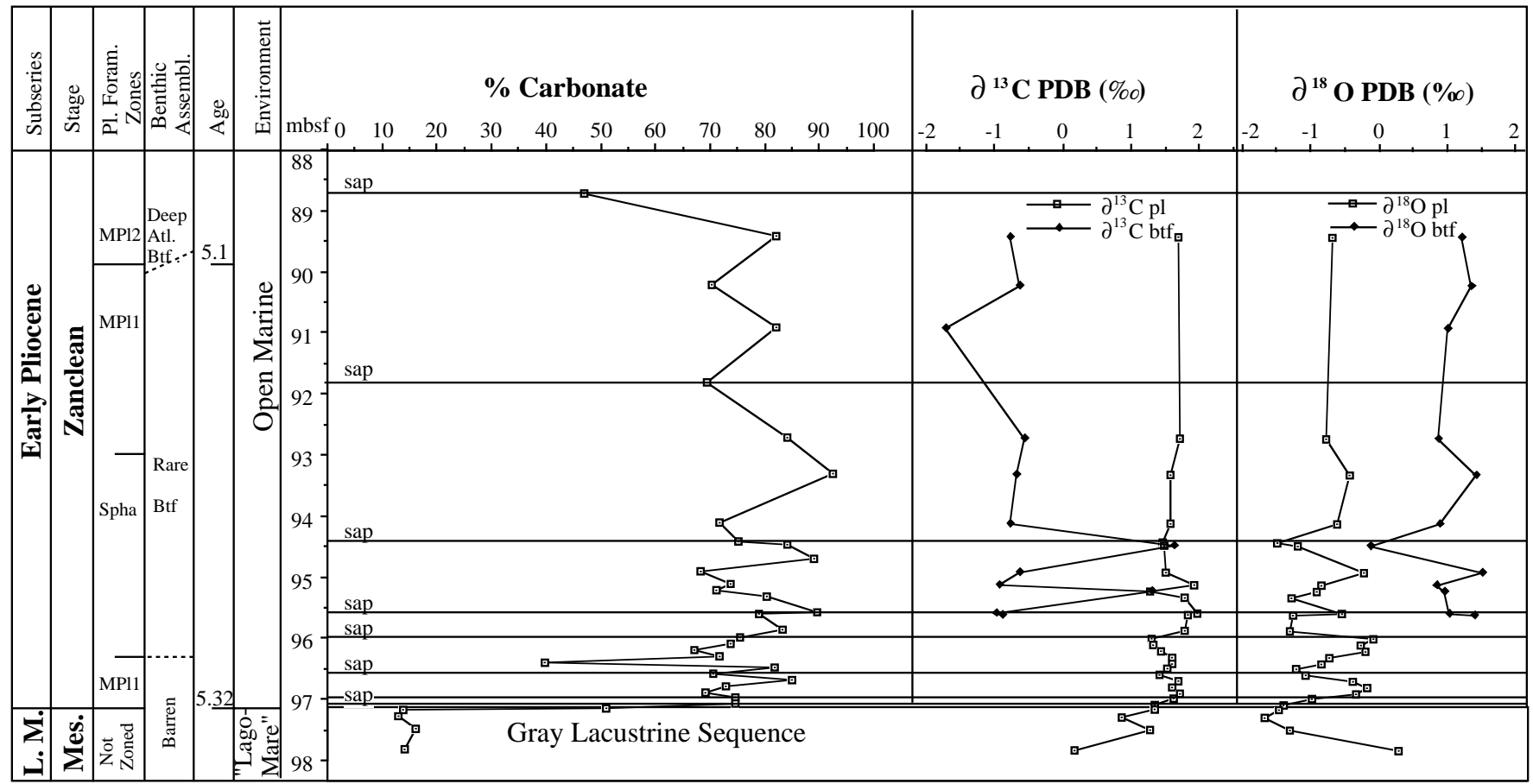

Spha = Sphaeroidinellopsis acme event

- Globigerinoides obliquus

- Oridorsalis stellatus

Figure 5. Summary of chemo- and biostratigraphy of the uppermost Miocene and lowermost Pliocene sediments of Hole 969B. Numerical age from Berggren et al. (1995). Horizontal lines (sap) indicate samples taken within sapropels.

ODP Leg 160 - Hole 969E

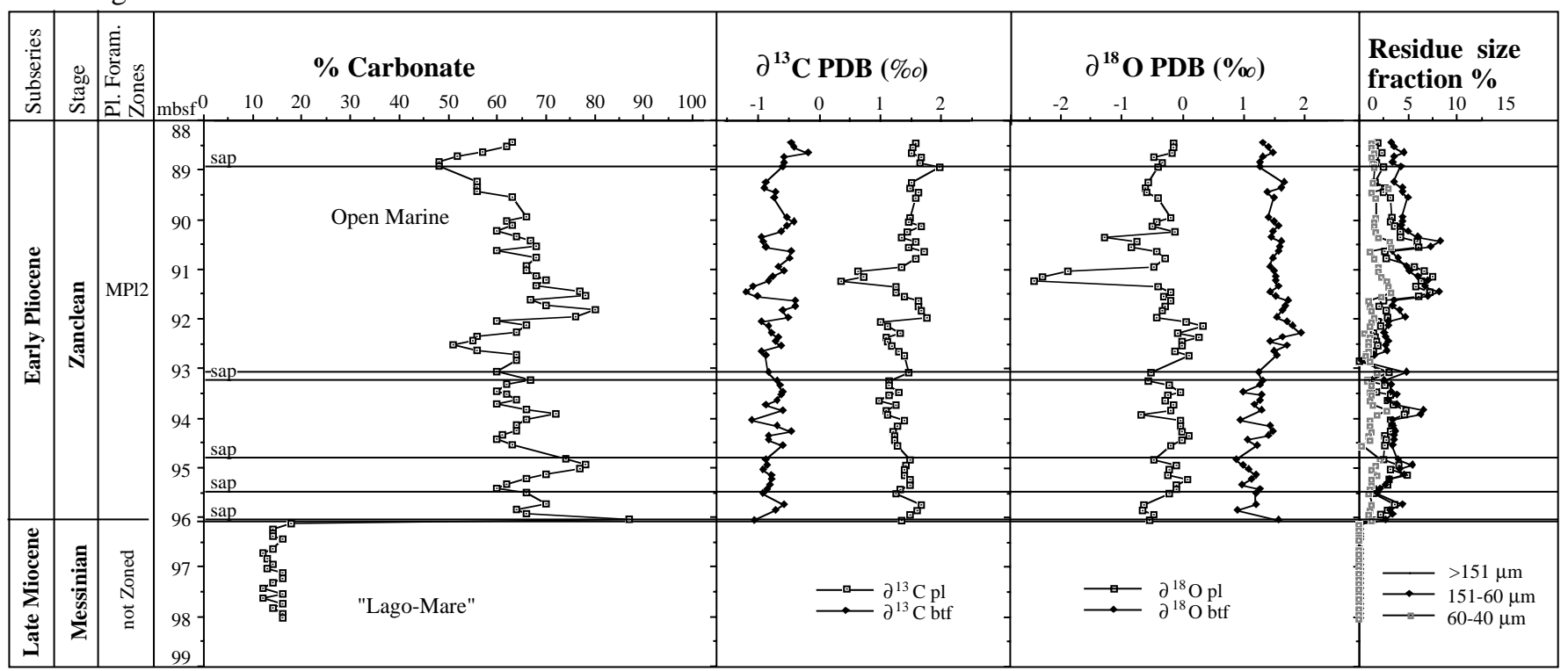

Figure 6. Summary of chemo- and biostratigraphy of the uppermost Miocene and lowermost Pliocene sediments of Hole 969E. Horizontal lines (sap) indicate samples taken within sapropels. 
ODP Leg 160 - Hole 967A

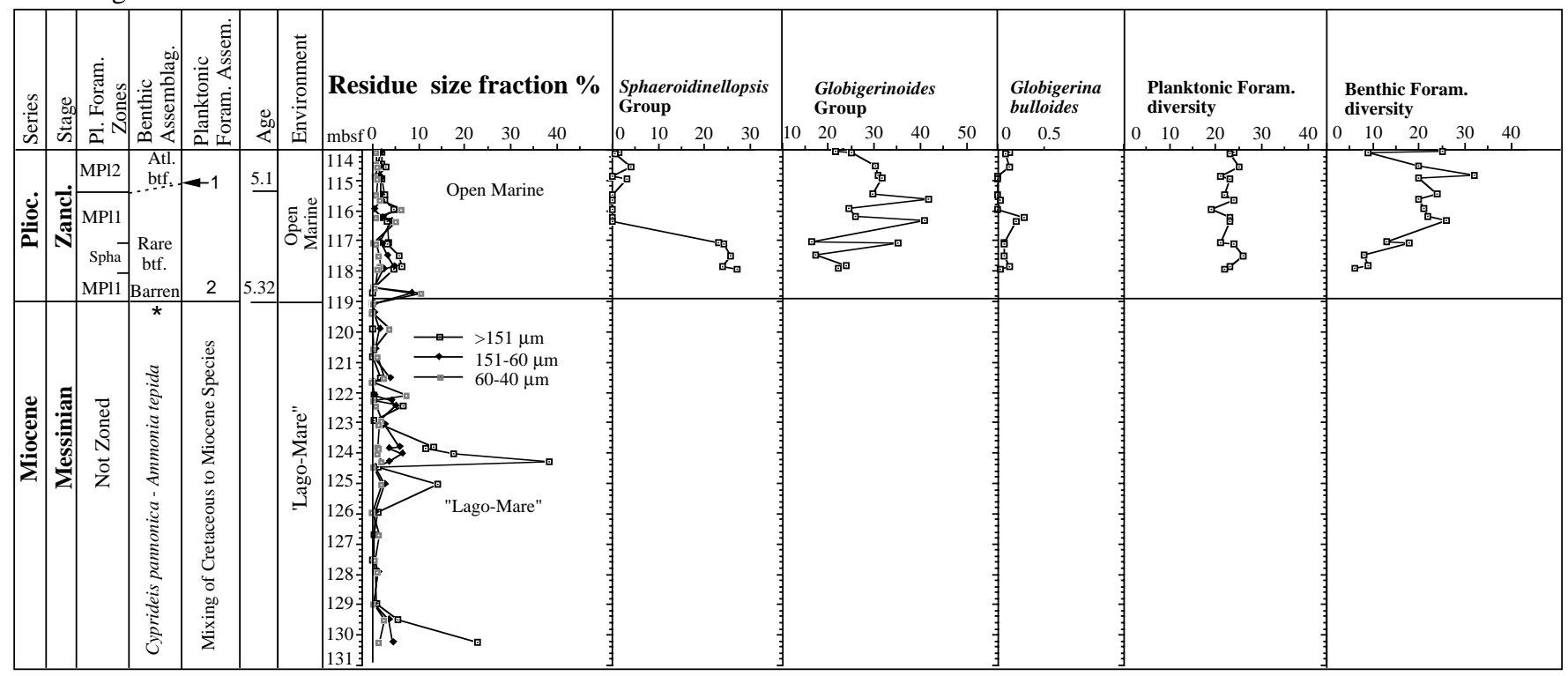

Figure 7. Plot of the abundance of Sphaeroidinellopsis, Globigerinoides groups, and Globigerina bulloides and planktonic and benthic foraminifer species abundance for Hole $967 \mathrm{~A}$.

Figure 8. Plot of the abundance of Sphaeroidinellopsis, Globigerinoides groups, and Globigerina bulloides and benthic foraminifer species abundance for Hole 969B.

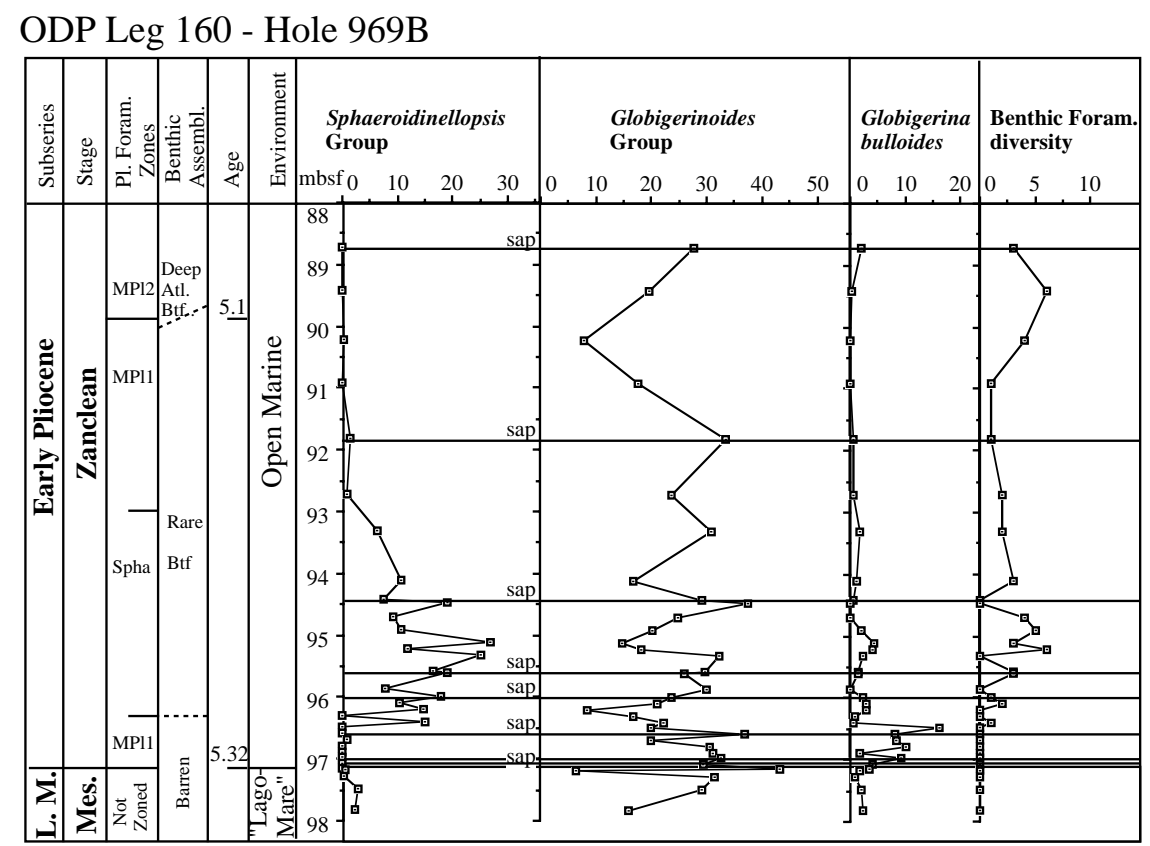

in the Eastern Mediterranean, the terminal Messinian flood and the earliest Pliocene paleoceanography appear to be similar and most likely synchronous throughout the entire Mediterranean Sea.

\section{ACKNOWLEDGMENTS}

We thank ODP for inviting Silvia Spezzaferri aboard on Leg 160 and providing the large number of samples. J. Terranes and S. Bernasconi are acknowledged for their assistance with the stable isotope measurements. The paper was helpfully reviewed by S. Iaccarino and R. Sprovieri. Financial support was given to Silvia Spezzaferri by the
European Science Foundation and the Italian Consiglio Nazionale Ricerche (CNR).

\section{REFERENCES}

Benson, R.H., 1978. The paleoecology of the ostracodes of DSDP Leg 42A. In Hsü, K.J., Montadert, L., et al., Init. Repts. DSDP, 42 (Pt. 1): Washington (U.S. Govt. Printing Office), 777-787.

Benson, R.H., and Hodell, D.A., 1994. Comment on "A critical re-evaluation of the Miocene/Pliocene boundary as defined in the Mediterranean" by F.J. Hilgen and C.G. Langereis. Earth Planet. Sci. Lett., 118:135-144.

Berggren, W.A., Hilgen, F.J., Langereis, C.G., Kent, D.V., Obradovich, J.D., Raffi, I., Raymo, M.E., and Shackleton, N.J., 1995. Late Neogene chro- 
nology: new perspectives in high-resolution stratigraphy. Geol. Soc. Am. Bull., 107:1272-1287.

Blechschmidt, G., Cita, M.B., Mazzei, R., and Salvatori, G. 1982. Stratigraphy of the Western Mediterranean and Southern Calabrian Ridges, Eastern Mediterranean. Mar. Micropaleontol., 7:101-134.

Cita, M.B., 1973a. Inventory of biostratigraphical finding and problems. In Ryan, W.B.F., Hsü, K.J., et al., 1973. Init. Repts. DSDP, 13: Washington (U.S. Govt. Printing Office), 1045-1074.

1973b. Pliocene biostratigraphy and chronostratigraphy. In Ryan, W.B.F., Hsü, K.J., et al. Init. Repts. DSDP, 13 (Pt. 2): Washington (U.S. Govt. Printing Office), 1343-1379.

, 1975a. The Miocene/Pliocene boundary: history and definition. In Saito, T., and Burckle, L.H., (Eds.), Late Neogene Epoch Boundaries. Spec. Publ. Micropaleontol., 1-30.

1975b. Studi sul Pliocene e gli strati di passaggio dal Miocene al Pliocene, VII. Planktonic foraminiferal biozonation of the Mediterranean Pliocene deep sea record: a revision. Riv. Ital. Paleontol. Stratigr., $81: 527-544$.

Cita, M.B., and Gartner, S., 1973. Studi sul Pliocene e sugli strati al passaggio dal Miocene al Pliocene. IV. The stratotype Zanclean foraminiferal and nannofossil biostratigraphy. Riv. Ital. Paleontol. Stratigr., 79:503558.

Cita, M.B., Ramil, C., Wright, C., Ryan, W.B.F., and Longinelli, A., 1978. Messinian paleoenvironment. In Hsü, K., Montadert, L., et al., Init. Repts. DSDP, 42A: Washington (U.S. Govt. Printing Office), 1003-1036.

Cita, M.B., and Ryan, W.B.F., 1973. Timescale and general synthesis. In Ryan, W.B.F., Hsü, K.J., et al., Init. Repts. DSDP; 13: Washington (U.S. Govt. Printing Office), 1405-1416.

Cita, M.B., Vismara-Schilling, A., and Bossio, A., 1980. Stratigraphy and paleoenvironment of the Cuevas del Almazora Section (Vera Basin): a reinterpretation. Riv. Ital. Paleontol. Stratigr., 86:215-240.

Craig, H., 1957. Isotopic standards for carbon and oxygen and correction factors for mass-spectrometric analysis of carbon dioxide. Geochim. Cosmochim. Acta, 12:133-149.

Emeis, K.-C., Robertson, A.H.F., Richter, C., et al., 1996. Proc. ODP, Init. Repts., 160: College Station, TX (Ocean Drilling Program).

Hasegawa, S., Sprovieri, R., and Poluzzi, A., 1990. Quantitative analyses of benthic foraminiferal assemblages from Plio-Pleistocene sequences in the Tyrrhenian Sea, ODP Leg 107. In Kastens, K.A., Mascle, J., et al., 1990. Proc. ODP, Sci. Results, 107: College Station, TX (Ocean Drilling Program), 461-478.

Hilgen, F.J., 1991. Extension of the astronomically calibrated (polarity) time scale to the Miocene/Pliocene boundary. Earth Planet. Sci. Lett., 107:349-368

Hilgen, F.J., and Langereis, C., 1993. A critical re-evaluation of the Miocene/ Pliocene boundary as defined in the Mediterranean. Earth Planet. Sci. Lett., 118:167-179.

Hsü, K.J., Cita, M.B., and Ryan, W.B.F., 1973. The origin of the Mediterranean evaporites. In Ryan, W.B.F., Hsü, K.J., et al., Init. Repts. DSDP, 13 (Pt. 2): Washington (U.S. Govt. Printing Office), 1203-1231.

Hsü, K.J., Montadert, L., et al., 1978. Init. Repts. DSDP, 42 (Pt. 1): Washington (U.S. Govt. Printing Office).

Iaccarino, S., 1985. Mediterranean Miocene and Pliocene planktic foraminifera. In Bolli, H.M., Saunders, J.B., and Perch-Nielsen, K. (Eds.), Plankton Stratigraphy: Cambridge (Cambridge Univ. Press), 283-314.

McKenzie, J.A., Hodell, D.A., Mueller, P.A., and Mueller, D.W., 1988. Application of strontium isotopes to late Miocene-early Pliocene stratigraphy. Geology, 16:1022-1025.

McKenzie, J.A., and Ricchiuto, T.E., 1978. Stable isotopic investigation of carbonate samples related to the Messinian salinity crisis from DSDP Leg 42A, Mediterranean Sea. In Hsü, K., Montadert, L., et al., Init. Repts. DSDP, 42A: Washington (U.S. Govt. Printing Office), 651-655.

McKenzie, J.A., and Sprovieri, R., 1990. Paleoceanographic conditions following the earliest Pliocene flooding of the Tyrrhenian Sea. In Kastens, K.A., Mascle, J., et al., Proc. ODP, Sci. Results, 107: College Station, TX (Ocean Drilling Program), 405-414.

McKenzie, J.A., Sprovieri, R., and Channell, J.E.T., 1990. The terminal Messinian flood and earliest Pliocene paleoceanography in the Mediterranean: results from ODP Leg 107, Site 652, Tyrrhenian Sea. Mem. Soc. Geol. Ital., 44:81-91.

Orszag-Sperber, F., and Rouchy, J.M., 1979. Le Miocene terminal et le Pliocene inferieur au Sud de Chypre. V Sem. sur le Messinien, PIGC 117, Envenment Geologiques a la limite Miocene-Pliocene, 40-47.
Premoli Silva, I., and Boersma, A., 1988. Atlantic Eocene planktonic foraminiferal historical biogeography and paleohydrographic indices. Palaeogeogr., Palaeoclimatol., Palaeoecol., 67:315-356.

1989. Atlantic Paleogene planktonic foraminiferal bioprovincial indices. Mar. Micropaleontol., 14:357-371.

Premoli Silva, I., Castradori, D., and Spezzaferri, S., 1993. Calcareous nannofossil and planktonic foraminifer biostratigraphy of Hole 810C (Shatsky Rise, northwestern Pacific). In Natland, J.H., Storms, M.A., et al., Proc. ODP, Sci. Results, 132: College Station, TX (Ocean Drilling Program), 15-36.

Ryan, W.B.F., 1973. Geodynamic implications in the Messinian crisis of salinity. In Messinian events in the Mediterranean. K. Nederl. Akad. Wetensh., 26-38.

Ryan, W.B.F., Hsü, K.J., et al., 1973. Init. Repts. DSDP, 13 (Pts. 1 and 2): Washington (U.S. Govt. Printing Office).

Spezzaferri, S., 1994. Planktonic foraminiferal biostratigraphy and taxonomy of the Oligocene and lower Miocene in the oceanic record: an overview. Palaeontographica Ital., 81:1-187.

1995. Planktonic foraminiferal paleoclimatic implications across the Oligocene-Miocene transition in the oceanic record (Atlantic, Indian and South Pacific). Palaeogeogr., Palaeoclimatol., Palaeoecol., 114:4374.

Sprovieri, R., 1992. Mediterranean Pliocene biochronology: a high resolution record based on quantitative planktonic foraminifera distribution. Riv. Ital. Paleontol. Stratigr., 98:61-100.

1993. Pliocene-early Pleistocene astronomically forced planktonic foraminifera abundance fluctuations and chronology of Mediterranean calcareous plankton bio-events. Riv. Ital. Paleontol. Stratigr. 99:371-414.

Sprovieri, R., and Hasegawa, S., 1990. Plio-Pleistocene benthic foraminifer stratigraphic distribution in the deep-sea record of the Tyrrhenian Sea (ODP Leg 107). In Kastens, K.A., Mascle, J., et al., Proc. ODP, Sci. Results, 107: College Station, TX (Ocean Drilling Program), 429-459.

Wright, R., 1980. Benthic foraminiferal repopulation of the Mediterranean after the Messinian (late Miocene) event. Palaeogeogr., Palaeoclimatol., Palaeoecol., 29:169-188.

Zijderveld, J.D.A., Zachariasse, W.J., Verhallen, P.J.J.M., and Hilgen, F.J., 1986. The age of the Miocene-Pliocene boundary. Newsl. Stratigr. 16:169-181.

\section{Date of initial receipt: 15 January 1997 \\ Date of acceptance: 1 August 1997 \\ Ms 160SR-026}

\section{APPENDIX}

\section{List of Species and Taxonomic Notes}

Pliocene Planktonic Foraminifers

Planktonic and benthic foraminiferal species are listed in alphabetical order by genus. The generic and specific concepts and the species groups used by Iaccarino (1985) and Spezzaferri (1994) are retained herein, whenever possible.

Beella digitata $($ Brady, 1879) (= Globigerina digitata Brady)

Globigerina bulbosa LeRoy, 1944

Globigerina bulloides d'Orbigny, 1926

Globigerina falconensis Blow, 1959

"Globigerina" venezuelana Hedberg, 1937

Globigerinella obesa (Bolli, 1957) (= Globorotalia obesa Bolli)

Globigerinella siphonifera (d'Orbigny, 1839) (= Hastigerina siphonifera d'Orbigny)

Globigerinita glutinata (Egger, 1893) (= Globigerina glutinata Egger)

Globigerinita juvenilis (Bolli, 1957) (= Globigerina juvenilis Bolli)

Globigerinita uvula (Ehrenberg, 1861) (= Pylodexia uvula Ehrenberg)

Globigerinoides gomitulus (Seguenza, 1880) (= Globigerina gomitulus Seguenza)

Globigerinoides obliquus Bolli, 1957

Globigerinoides quadrilobatus (d'Orbigny, 1846) (= Globigerina quadrilobata d'Orbigny)

Globigerinoides ruber (d'Orbigny, 1839) (= Globigerina rubra d'Orbigny) 
Globigerinoides sacculifer (Brady, 1877) (= Globigerina sacculifera Brady) Globigerinoides trilobus (Reuss, 1850) (= Globigerina triloba Reuss)

Globorotalia margaritae Bolli and Bermudez, 1965

Globorotalia menardii (Parker, Jones, and Brady, 1865) (= Rotalia menardii Parker, Jones, and Brady)

Globorotalia scitula (Brady, 1882) (= Pulvinulina scitula Brady)

Neogloboquadrina acostaensis (Blow, 1959) (= Globorotalia acostaensis Blow)

Neogloboquadrina dutertrei (d'Orbigny, 1839) (= Globigerina dutertrei d'Orbigny)

Neogloboquadrina pachyderma (Ehrenberg, 1861) (= Aristospira pachyderma Ehrenberg)

Orbulina universa d'Orbigny, 1839

Sphaeroidinella dehiscens (Parker and Jones, 1865) (= Sphaeroidina bulloides d'Orbigny var. dehiscens Parker and Jones)

Sphaeroidinellopsis kochi (Caudri, 1934) (= Globigerina kochi Caudri)

Sphaeroidinellopsis subdehiscens Blow, 1969

Sphaeroidinellopsis seminulina (Schwager, 1866) (= Globigerina seminulina Schwager)

Tenuitella anfracta (Parker, 1967) (= Globorotalia anfracta Parker)

Turborotalita quinqueloba Natland, 1938 (= Globigerina quinqueloba, Natland)

Zeaglobigerina decoraperta (Takayanagi and Saito, 1962) (= Globigerina decoraperta Takayanagi and Saito)

Zeaglobigerina nepenthes (Todd, 1957) (= Globigerina nepenthes Todd). Small-sized specimens with smaller aperture than typical $Z$. nepenthes and resembling Zeaglobigerina druryi have been separated under Z. nepenthes group.

Zeaglobigerina woodi (Jenkins, 1960) (= Globigerina woodi Jenkins)

Streptochilus globigerum (Schwager, 1986) (= Textilaria globigera Schwager)

Planktonic Foraminifers Contained in the Messinian Sediments

The generic and specific concepts and the species groups are those used by Premoli Silva and Boersma $(1988,1989)$ and Spezzaferri (1994).

Acarinina bullbrooki (Bolli, 1957) (= Globorotalia bullbrooki Bolli)

Acarinina pentacamerata Subbotina, 1953

Acarinina soldadoensis (Brönnimann, 1952) (= Globigerina soldadoensis Brönnimann)

Cassigerinella chipolensis (Cushman and Ponton, 1932) (= Cassidulina chipolensis Cushman and Ponton)

Chiloguembelina cubensis (Palmer, 1934) (= Guembelina cubensis Palmer).

Dentoglobigerina galavisi (Bermudez, 1961) (= Globigerina galavisi Bermudez)

"Globigerina" ciperoensis Bolli, 1957

Globoquadrina dehiscens (Chapman, Parr and Collins, 1934) (= Globorotalia dehiscens Chapman, Parr and Collins)

Paragloborotalia pseudokugleri (Blow, 1969) (= Globorotalia pseudokugleri Blow)

Paragloborotalia acrostoma (Wezel, 1966) (= Globorotalia acrostoma Wezel)

Paragloborotalia kugleri (Bolli, 1957) (= Globorotalia kugleri Bolli)

Paragloborotalia opima (Bolli, 1957) (= Globorotalia opima opima Bolli)

Planorotalites pseudoscitulus (Glaessner, 1937) (= Globorotalia pseudoscitula Glaessner)

Praeorbulina transitoria (Blow, 1956) (= Globigerinoides transitoria Blow)

Subbotina angiporoides angiporoides (Hornibrook, 1965) (= Globigerina angiporoides Hornibrook)

Subbotina utilisindex (Jenkins and Orr, 1973) (= Globigerina utilisindex Jenkins and Orr)

Turborotalia griffinae Blow, 1979

Zeaglobigerina ampliapertura (Bolli, 1957) (= Globigerina ampliapertura Bolli)

Benthic Foraminifers

Generic and specific concepts of Sprovieri and Hasegawa (1990) and Hasegawa et al. (1990) are retained here.
Anomalinoides helicinus (Costa, 1857) (= Nonionina helicina Costa)

Astrononion stelligerum (d'Orbigny, 1839) (= Nonionina stelligera d'Orbigny)

Bigenerina nodosaria d'Orbigny, 1826

Bolivina subspinescens Cushman, 1922

Brizalina punctata d'Orbigny, 1839

Brizalina dilatata (Reuss, 1850) (= Bolivina dilatata Reuss)

Cibicidoides italicus (Di Napoli, 1952) (= Cibicides italicus Di Napoli)

Cibicidoides ornatus $($ Costa, 1856) (= Nonionina ornata Costa)

Cibicidoides pachyderma (Rzehak, 1866) (= Truncatulina pachyderma Rzehak)

Cibicidoides ungerianus (d'Orbigny, 1846) (= Rotalina ungeriana d'Orbigny)

Cribrobulimina serpens Seguenza emend. Sellii, 1941

Cylindroclavulina rudis (Costa, 1855) (= Glandulina rudis Costa)

Dentalina leguminiformis (Batsch, 1791) (= Nautilus leguminiformis Batsch)

Eggerella bradyi (Cushman, 1911) (= Verneuilina brady Cushman)

Globocassidulina subglobosa (Brady, 1881) (= Cassidulina globosa Brady)

Gyroidina soldanii (d'Orbigny, 1826) (= Rotalina soldanii d'Orbigny)

Gyroidinoides laevigataus (d'Orbigny 1826) (= Gyroidina laevigata d'Orbigny)

Karreriella bradyi (Cushman, 1911) (= Gaudryna brady Cushman)

Lagena clavata (d'Orbigny, 1846) (Oolina clavata d'Orbigny)

Lenticulina cultrata (de Montfort, 1808) (= Robulus cultratus de Montfort)

Lenticulina gibba (d'Orbigny, 1839) (= Cristellaria gibba d'Orbigny)

Lenticulina inornata (d'Orbigny, 1846) (= Robulina inornata d'Orbigny)

Lenticulina rotulata (Lamark, 1804) (= Lenticulites rotulata Lamark)

Lobatula lobatula (Walter and Jacob, 1798) (= Nautilus lobatulus Walter and Jacob 1798)

Martinottiella communis (d'Orbigny, 1826) (= Clavulina communis d'Orbigny)

Martinottiella perparva (Cushman, 1936) (= Listerella communis (d'Orbigny) var. perparva Cushman)

Oolina faveolata (Seguenza, 1862) (= Orbulina faveolata Seguenza)

Oridorsalis stellatus (Silvestri, 1898) (= Truncatulina tenera Brady var. stellata Silvestri)

Parrelloides bradyi (Trauth, 1918) -Parrelloides robertsonianus (Brady, 1881). According with Sprovieri and Hasegawa (1990) these two forms are here considered as macrospheric and microspheric forms of the same species. However, in the range chart the two morphotypes are separate because of the distinctive characters of $P$. bradyi displaying smaller size, reduced numbers of chambers in the last whorl and oblique sutures on the spiral side.

Planulina ariminensis d'Orbigny, 1826

Pleurostomella alternans Schwager, 1866

Pseudoparrella exigua Conato, 1964

Pullenia bulloides (d'Orbigny, 1846) (= Nonionina bulloides d'Orbigny)

Pullenia osloensis Feyling-Hanssen, 1954

Pullenia quadriloba (Reuss, 1851) (= Nonionina quadriloba Reuss)

Pullenia quinqueloba (Reuss, 1851) (= Nonionina quadriloba Reuss)

Pullenia salisbury Stewart and Stewart, 1930

Quinqueloculina bicarinata d'Orbigny, 1826

Quinqueloculina laevigata. d'Orbigny, 1949

Quinqueloculina viennensis J. and Y. Le Calvez, 1958

Sigmoilinita tenuis (Czjek, 1848) (= Quinqueloculina tenuis $\mathrm{Czjek)}$

Sigmoilopsis schlumbergeri (Silvestri, 1904) (= Sigmoilina schlumbergeri Silvestri)

Siphonina planoconvexa (Silvestri, 1898) (= Truncatulina reticulata $($ Czjek) var. planoconvexa Silvestri)

Siphonina reticulata (Czjek, 1848) (= Rotalina reticulata Czjek)

Sphaeroidina bulloides d'Orbigny, 1826

Stilostomella antillea (Cushman, 1923) (= Nodosaria antillea Cushman)

Stilostomella hispida (d'Orbigny) (= Nodosaria hispida d'Orbigny)

Stilostomella monilis (Silvestri, 1872) (= Nodosaria monilis Silvestri)

Uvigerina peregrina Cushman, 1923

Uvigerina pygmaea d'Orbigny, 1826

Uvigerina rutila Cushman and Todd, 194

Vaginulinopsis carinata $($ Silvestri, 1904) $(=$ Vaginulinopsis inversa $($ Costa) var. carinata Silvestri

Valvulineria marmorea Conato, 1964

Vulvulina pennatula (Batsch, 1791) (= Nautilus pennatulus Batsch) 


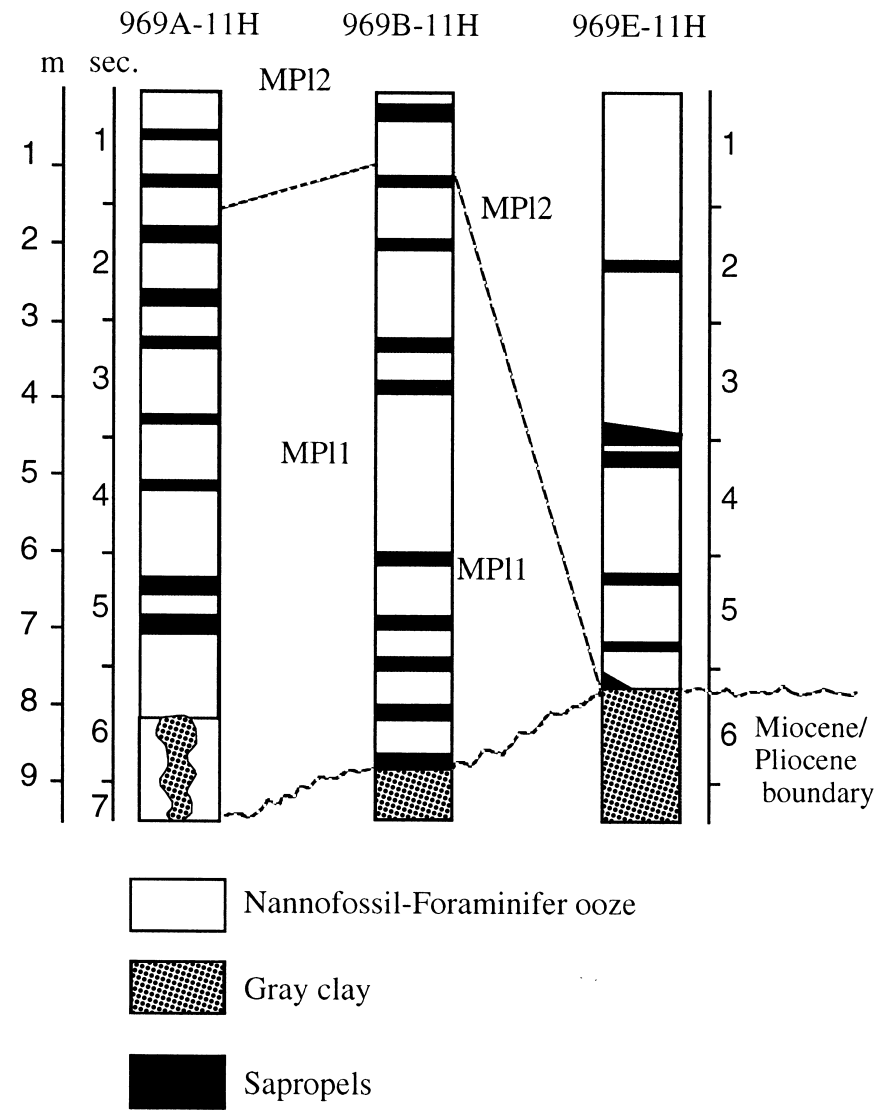

Figure 9. Correlation of Cores 160-969A-11H, 969B-11H and 969E-11H. Note that the most complete sequence is from Hole 969B. 


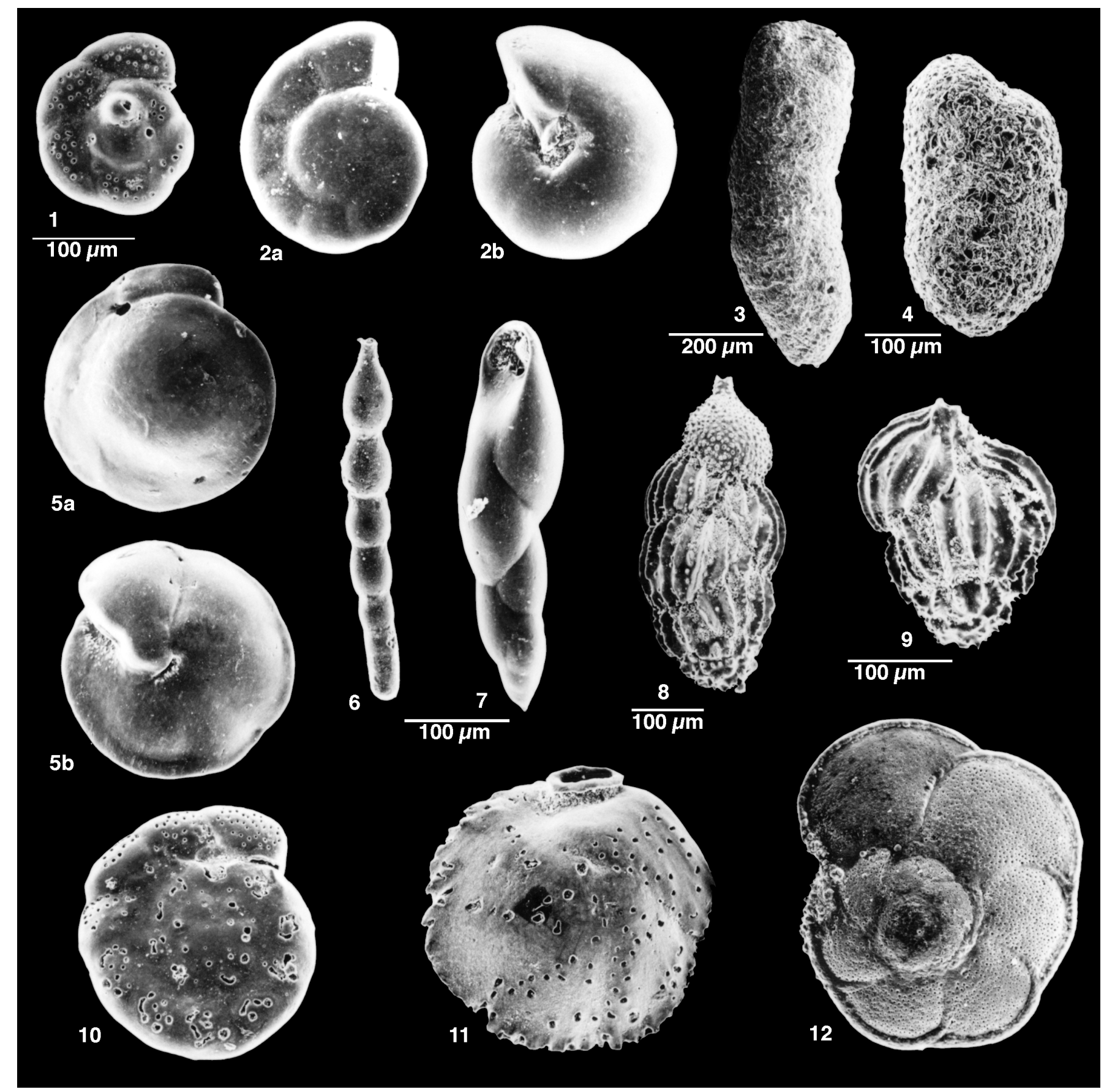

Plate 1. Benthic and the planktonic foraminifer Globorotalia menardii from the Eastern Mediterranean. 1. Parrelloides bradyi (Trauth), Sample 160-967A-12H1, 65-67 cm. 2a-b. Gyroidinoides laevigatus (d'Orbigny), Sample 160-967A-12H-1, 65-67 cm. 3. Martinottiella perparva (Cushman), Sample 160-167A13H-2, 77-79 cm. 4. Martinottiella communis (d'Orbigny), Sample 160-967A-12H-1, 65-67 cm. 5a-b. Oridorsalis stellatus (Silvestri), Sample 160-967A12H-1, 65-67 cm. 6. Stilostomella antillea (Cushman), Sample 160-967A-12H-1, 65-67 cm. 7. Pleurostomella alternans Schwager, Sample 160-967A-12H-1, 65-67 cm. 8. Uvigerina pygmaea d'Orbigny, Sample 160-967A-12H-1, 65-67 cm. 9. Uvigerina cf. peregrina Cushman, Sample 160-967A-12H-1, 65-67 cm. 10. Parrelloides robertsonianus (Brady), Sample 160-967A-12H-1, 65-67 cm. 11. Siphonina reticulata (Czjek), Sample 160-967A-12H-1, 65-67 cm. 12. Globorotalia menardii (Parker, Jones, and Brady), Sample 160-967A-13H-2, 35-37 cm. (a = spiral view, b = umbilical view). Figures 1, 2, 5, 6, 7, 10, 11 and 12 are at the same magnification. 


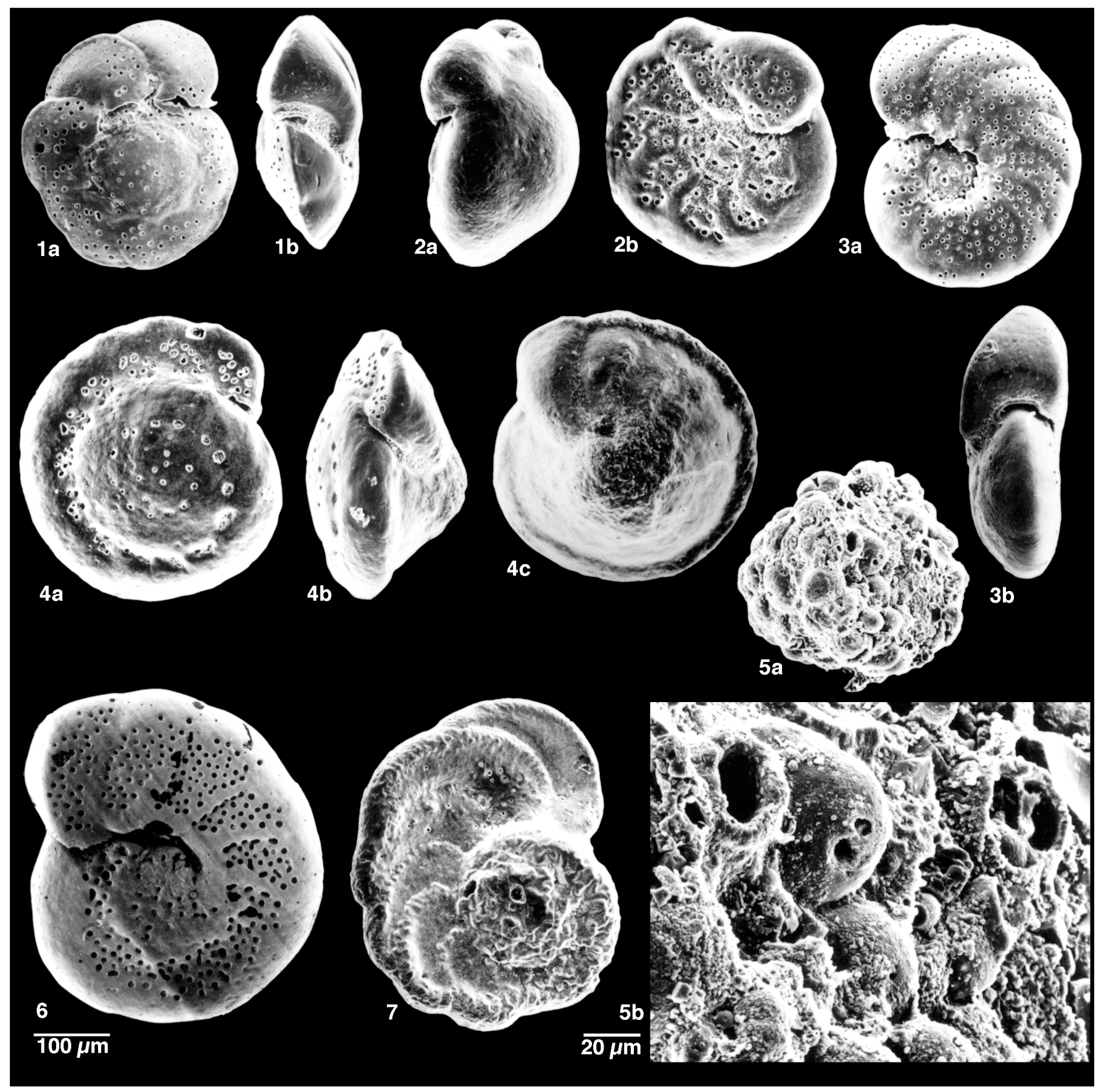

Plate 2. Benthic foraminifer from the Eastern Mediterranean. 1a-b. Cibicidoides ungerianus (d'Orbigny), Sample 160-167A-13H-2, 77-79 cm. 2a-b. Cibicidoides italicus (Di Napoli), Sample 160-967A-12H-1, 65-67 cm, a = side view and b = umbilical view. 3a-b. Anomalinoides helicinus (Costa), Sample 160967A-12H-1, 65-67 cm. 4a-c. Cibicidoides pachyderma (Rzehak), Sample 160-967A-12H-1, 65-67 cm. 5a-b. Cylindroclavulina rudis (Costa), Sample 16067A-12H-5, 61-63 cm, b, detail of the wall texture. 6. Cibicidoides ornatus (Costa), Sample 160-967A-12H-5, 61-63 cm, spiral view. 7. Planulina ariminensis d'Orbigny, Sample 160-967A-12H-1, 65-67 cm, spiral view. ( $\mathrm{a}=$ spiral view, $\mathrm{b}=$ side view, $\mathrm{c}=$ umbilical view). All specimens except 5b are at the same magnification. 


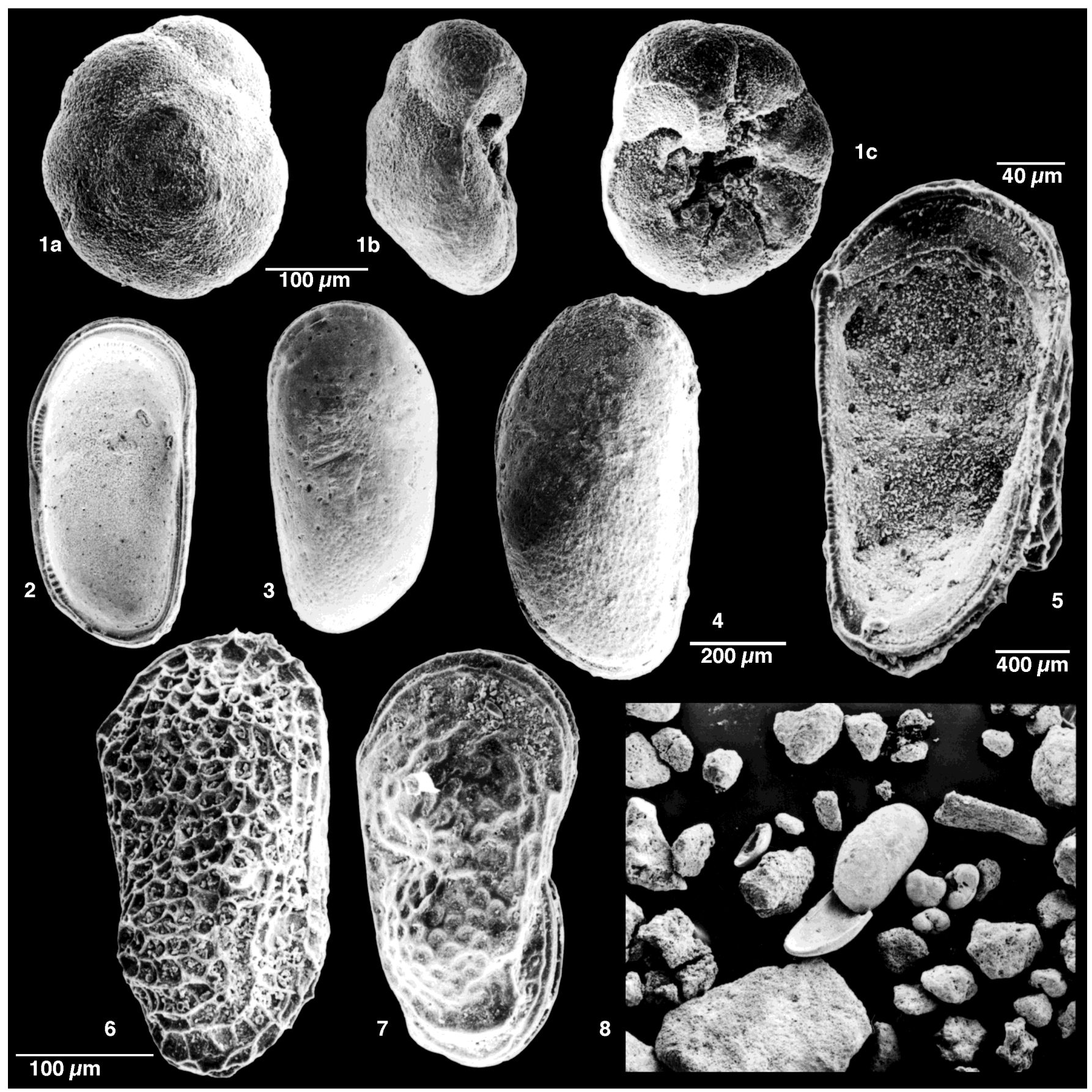

Plate 3. Lago Mare fauna and facies in the Eastern Mediterranean. 1a-c. Ammonia tepida Cushman. $\mathrm{a}=$ spiral view, $\mathrm{b}=$ side view, $\mathrm{c}=$ umbilical view. 2. Cyprideis pannonica (Mehes), Sample 160-967A-13H-7, 12-14 cm. 3. Cyprideis pannonica (Mehes), Sample 160-967A-14H-1, 50-52 cm. 4. Cyprideis pannonica (Mehes), specimen with the valves still attached, Sample 160-967A-13H-7, 12-14 cm. 5. Loxoconca diaffarovi Schneider, Sample 160-967A-13H-4, 109-111 $\mathrm{cm}$. 6. Loxoconca diaffarovi Schneider, specimen with the valves still attached, Sample 160-967A-13H-4, 109-111 cm. 7. Loxoconca diaffarovi Schneider, specimen with the valves still attached, Sample 160-967A-13H-4, 109-111 cm. 8. Lago Mare facies. Note the specimens of C. pannonica and A. tepida, Sample 160-967A-14X-2, 21-23 cm. Figures 2, 3, 4, 6, and 7 are at the same magnification. 